\title{
Rationale Klimapolitik - ökonomische Anforderungen und politische Hindernisse
}

\author{
Rupert Pritzl · Fritz Söllner (iD
}

Angenommen: 21. April 2021 / Online publiziert: 6. Mai 2021

(C) Der/die Autor(en) 2021

Zusammenfassung In der klimapolitischen Debatte stehen meist einzelne Instrumente und Maßnahmen im Vordergrund, wohingegen die Frage der ökonomischen Rationalität der Klimapolitik als solcher häufig vernachlässigt wird. Eine Klimapolitik wäre dann ökonomisch rational, wenn politisch vorgegebene Klimaziele mit geringstmöglichen Kosten realisiert werden würden. Notwendig hierfür ist eine umfassende und einheitliche Bepreisung der Treibhausgasemissionen. Dieser rationalen Klimapolitik wird die aktuelle deutsche und europäische Politik gegenübergestellt, die sich als ineffektiv und ineffizient erweist. Ursächlich hierfür sind die Anreize, denen die politischen Akteure unterliegen und die zu einem Widerspruch zwischen ökonomischer Rationalität und politischer Opportunität führen.

Schlüsselwörter Klimawandel · Klimapolitik · Ökonomische Rationalität · Politische Opportunität · Deutsche Klimapolitik · Green Deal EU

\footnotetext{
R. Pritzl

Bayerisches Staatsministerium für Wirtschaft, Landesentwicklung und Energie, München,

Deutschland

E-Mail: rupert.pritzl@stmwi.bayern.de

F. Söllner $(\bowtie)$

Technische Universität Ilmenau, Ilmenau, Deutschland

E-Mail: fritz.soellner@tu-ilmenau.de
} 


\title{
Rational climate policy_economic demands and political obstacles
}

\begin{abstract}
The debate about climate policy is mainly concerned with particular measures and instruments. Only rarely is the economic rationality of climate policy as such discussed. Climate policy would be economically rational, if climate objectives, which are to be determined politically, are realized with minimum costs. To this end, a comprehensive and uniform price on the emission of greenhouse gases has to be established. The actual climate policy, in Germany and in Europe, is far from being economically rational: It is both ineffective and inefficient. This is due to political incentives which make inefficient policies politically more attractive than efficient ones.
\end{abstract}

Keywords Climate change $\cdot$ Climate policy $\cdot$ Economic rationality $\cdot$ Political opportunism · German climate policy · Green Deal EU

\section{Klimapolitik und ökonomische Rationalität}

In der wissenschaftlichen und in der öffentlichen Diskussion der Klimapolitik stehen überwiegend einzelne klimapolitische Maßnahmen im Vordergrund. So wird die Sinnhaftigkeit des Kohleausstiegs thematisiert; die Effektivität und die Effizienz des Erneuerbare-Energien-Gesetzes werden kritisch hinterfragt; die Rolle negativer Emissionstechnologien wird erörtert; oder die Art und Weise der Förderung der Elektromobilität wird kritisiert. ${ }^{1}$ Diese und ähnliche Fragestellungen sind zwar vollkommen berechtigt, aber es besteht dabei die Gefahr, den Blick auf das ,große Ganze“ zu verlieren und die Einordnung all dieser Maßnahmen in eine systematische klimapolitische Strategie zu vernachlässigen. Aus diesem Grund wollen wir im Folgenden versuchen, die Frage zu beantworten, wie eine rationale Klimapolitik prinzipiell angelegt sein muss. Dabei geht es uns weniger um diese oder jene Einzelmaßnahme, sondern um den grundlegenden klimapolitischen Rahmen, in den sich die verschiedenen Einzelmaßnahmen einfügen müssen, wenn eine rationale Klimapolitik verwirklicht werden soll.

Unter Rationalität soll der Rationalitätsbegriff der Ökonomie verstanden werden, also die Zweckrationalität. Ökonomisch rational wäre die Klimapolitik folglich dann, wenn bestimmte Ziele bestmöglich erreicht werden können. Dies impliziert auf jeden Fall eine systematische und konsistente Herangehensweise. „Bestmöglich“ wird in der Ökonomie meist als „,mit den geringstmöglichen Kosten“, also im Sinn der Kosteneffizienz, interpretiert; daneben können aber auch Kriterien wie die Wahrscheinlichkeit und die Schnelligkeit der Zielerreichung eine Rolle spielen. Die Ziele selbst können entweder außerökonomisch, also politisch, vorgegeben sein oder aus dem ökonomischen Oberziel der Wohlfahrtsmaximierung mittels wohlfahrtsökonomische Methoden abgeleitet werden.

Wir verfolgen in diesem Beitrag den erstgenannten Ansatz und unterstellen ein auf der Grundlage naturwissenschaftlicher Erkenntnisse politisch vorgegebenes Kli-

\footnotetext{
1 Vgl. zu diesen Themen z.B. Cassel (2017), Rickels et al. (2019) und Weimann (2019, 2020).
} 
maziel - nämlich das auf der UN-Klimakonferenz von Paris im Dezember 2015 vereinbarte Ziel, die Erderwärmung bis zum Jahr 2050 auf deutlich unter $2,0^{\circ} \mathrm{C}$, möglichst sogar unter $1,5^{\circ} \mathrm{C}$ im Verhältnis zum vorindustriellen Niveau zu begrenzen und langfristig Klimaneutralität sicherzustellen. Welche Politik sollte aus Sicht der Ökonomie zur Realisierung dieses Ziels verfolgt werden? Die Beantwortung dieser Frage steht im Mittelpunkt des vorliegenden Beitrags, der auch die aktuelle deutsche bzw. europäische Klimapolitik an diesem Maßstab misst.

\section{Das Klimaproblem aus naturwissenschaftlicher und ökonomischer Sicht}

Der Schutz des Klimas bleibt - auch in der aktuell von Corona beherrschten Zeit eine große globale Herausforderung. Der Klimawandel ist ein weltweites Phänomen, das alle Menschen weltweit betrifft. Er zeigt sich immer deutlicher in einem Anstieg der globalen Durchschnittstemperatur, in stärkeren Klimaschwankungen und häufigeren meteorologischen Extremen wie Stürmen, Dürren oder Hitzesommern. Seit dem Jahr 1900 ist die weltweite mittlere Temperatur um $1,1^{\circ} \mathrm{C}$ gestiegen. Als Hauptursache für diesen Temperaturanstieg sieht der ,Weltklimarat“ IPCC (Intergovernmental Panel on Climate Change) die mit der Industrialisierung einhergehende Emission von Treibhausgasen in die Atmosphäre (vor allem Kohlenstoffdioxid $\left(\mathrm{CO}_{2}\right)$, daneben auch Methan $\left(\mathrm{CH}_{4}\right)$, Lachgas $\left(\mathrm{N}_{2} \mathrm{O}\right)$ und fluorierte Gase) und spricht daher von einem anthropogenen Treibhauseffekt (Intergovernmental Panel on Climate Change 2016). Mit großer Wahrscheinlichkeit würde die fortgesetzte Emission von Treibhausgasen in der bisherigen Größenordnung zu einem weiteren Temperaturanstieg um ca. $2^{\circ} \mathrm{C}$ bis zum Ende dieses Jahrhunderts führen. Über diese Zusammenhänge besteht in der Wissenschaft ein weitgehender Konsens, wenngleich es Unklarheiten über die Rolle anderer klimarelevanter Faktoren (wie etwa die Sonnenaktivität oder kosmische Strahlung) und teils relativ große Unschärfen in den Klima-Simulationsmodellen gibt (Pindyck 2020). Zudem ist die vor allem aus dem Selbstverständnis der Wissenschaften heraus problematische Nähe von Klimapolitik und Klimaforschung kritisch zu hinterfragen (v. Storch und Krauß 2013). Dennoch erscheint es im Sinne des Vorsichtsprinzips vernünftig, den gegenwärtigen Stand der Klimaforschung zur Grundlage klimapolitischer Entscheidungen zu machen. Denn der vorhergesagte weitere Anstieg der Temperatur hätte wahrscheinlich gravierende Konsequenzen zur Folge, die von einer Zerstörung von Ökosystemen und einer Zunahme von Umweltkatastrophen über die Gefährdung der Nahrungsmittelversorgung und der menschlichen Gesundheit bis zu einem verstärkten Migrationsdruck und militärischen Konflikten um natürliche Ressourcen reichen (vgl. z. B. Edenhofer und Jakob 2017, S. 39-41). Um diese Entwicklung zu verhindern und um mit den schon jetzt unvermeidbaren Folgen des Klimawandels besser fertig werden zu können, wird es vom IPCC für notwendig gehalten, den Gesamttemperaturanstieg auf $2,0^{\circ} \mathrm{C}$, wenn möglich auf $1,5 \mathrm{Grad}$, zu begrenzen. Diesem Ziel hat sich die Staatengemeinschaft am 12. Dezember 2015 durch das Pariser Abkommen verschrieben, in dem festgelegt wurde, dass ,der Anstieg der durchschnittlichen Erdtemperatur deutlich unter $2{ }^{\circ} \mathrm{C}$ über dem vorindustriellen Niveau gehalten und Anstrengungen 
unternommen werden, um den Temperaturanstieg auf $1,5^{\circ} \mathrm{C}$ über dem vorindustriellen Niveau zu begrenzen“ (Artikel 2). Will man das ambitioniertere 1,5-GradZiel erreichen, so müssten die anthropogenen Treibhausgasemissionen bis zum Jahr 2030 um 45\% gegenüber dem Jahr 2010 sinken und ab 2050 dürfte es gar keine Nettoemissionen von Treibhausgasen mehr geben. Es dürften dann also nur noch so viele Treibhausgase ausgestoßen werden, wie ohne einen weiteren Anstieg der Konzentration derselben in der Atmosphäre absorbiert werden können (Treibhausgasbzw. Klimaneutralität) (Deutsche Energie-Agentur 2020, S. 10-17).

\section{Die Ziele der Klimapolitik}

Akzeptiert man das 1,5- bzw. 2,0-Grad-Celsius-Ziel, dann besteht die Aufgabe der Klimapolitik darin, dieses Ziel zu den geringstmöglichen Kosten zu erreichen. Da die globale Durchschnittstemperatur nicht direkt beeinflusst werden kann, sind die genannten Temperaturvorgaben in einem ersten Schritt in Ziele für die Treibhausgaskonzentration in der Atmosphäre und in einem zweiten Schritt in Ziele für die Emission von Treibhausgasen zu ,übersetzen“. Diese Ziele beziehen sich auf die Netto- und nicht auf die Bruttoemissionen von Treibhausgasen (vgl. Teil 4.2). Die „Übersetzung“ muss auf der Grundlage natur- bzw. klimawissenschaftlicher Erkenntnisse erfolgen. Der ökonomischen Analyse zugänglich und mithin das unmittelbare Ziel der Klimapolitik ist daher die Realisation einer bestimmten Menge an Treibhausgasemissionen bzw. eines bestimmten Emissionspfads. Entscheidend ist dabei, dass dieses Ziel ein globales sein muss, da auch die Treibhausgaskonzentration eine globale Größe ist und es deshalb keine Rolle spielt, von wem und wo Treibhausgase emittiert werden - sondern nur, welche Menge an Treibhausgasen insgesamt emittiert wird.

Ist es sinnvoll, dass sich einzelne Länder im Interesse des Klimaschutzes ambitioniertere nationale Emissionsziele setzen und unabhängig vom Verhalten der anderen Länder als Vorreiter vorangehen? Schaut man sich die Zahlen zu den weltweiten Treibhausgasemissionen an, so wird schnell klar, dass die EU oder gar Deutschland allein bei weitem nicht in der Lage sind, für eine spürbare Reduktion der Gesamtemissionen und damit für eine Stabilisierung des Weltklimas zu sorgen (siehe Abb. 1). Im Jahr 2017 betrug laut Bundesministerium für Umwelt der Anteil der EU (ohne Deutschland) an den weltweiten Treibhausgasemissionen lediglich ca. $8 \%$ (Deutschland hat einen Anteil von ca. 2\%). Zum Vergleich dazu betrug der Anteil der USA ca. $14 \%$ und der von China ca. $28 \%$. Angesichts der absoluten Zunahme an Treibhausgasemissionen in China und Indien sinkt der Anteil der EU und Deutschlands Jahr für Jahr. Ob die EU oder Deutschland sich ambitioniertere Emissionsziele setzt und diese - im günstigsten Fall - auch erreicht, spielt auf globaler Ebene keine Rolle dafür, ob es gelingt, den gegenwärtigen Trend der Erwärmung zu stoppen oder gar umzukehren (Edenhofer et al. 2020, S. 13; SVR 2019, S. 34).

Die Vorstellung, dass ein Land beim Klimaschutz vorangeht und die anderen Länder ihm folgen werden, ignoriert die grundlegenden ökonomischen Zusammenhänge: Je klimapolitisch aktiver ein Land ist, desto geringer ist der Anreiz für andere Länder, selbst eine teure Klimapolitik zu betreiben, und desto größer ist der Anreiz, 
Abb. 1 Länderanteile an globalen THG-Emissionen 2017. (Quelle: eigene Darstellung nach Daten aus BMU 2020, S. 12)

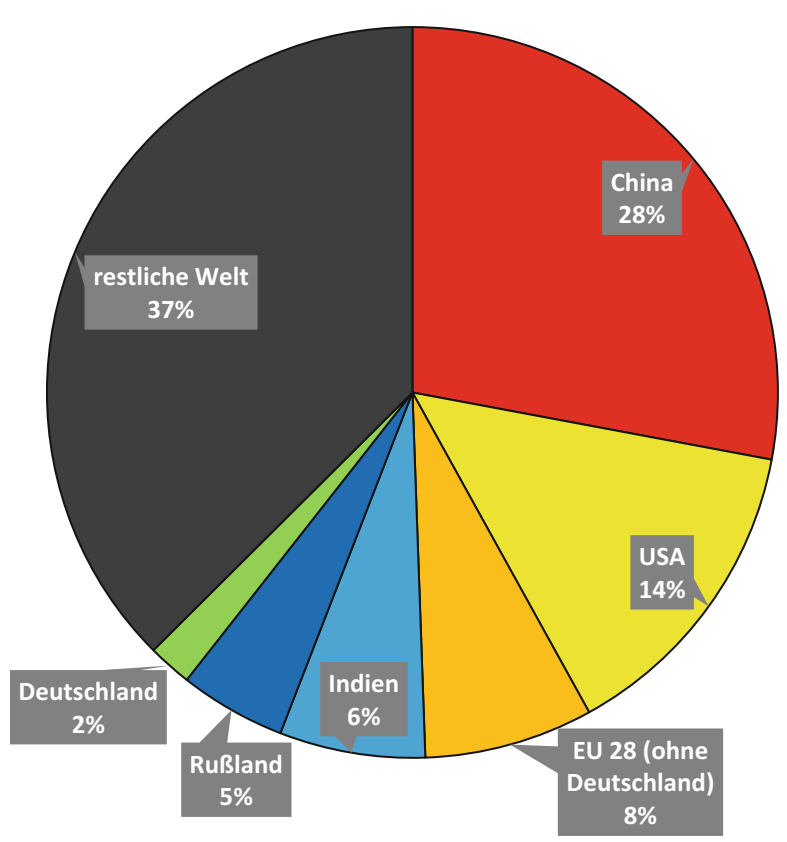

abzuwarten und klimapolitisch nichts zu tun - da ja die Trittbrettfahrerstrategie erfolgreich zu sein scheint. Das Kyoto-Protokoll aus dem Jahr 1997 lehrt uns, dass ein erster Versuch von Vorreiterländern, mit einseitigen Maßnahmen andere zum Mitmachen zu überzeugen, gescheitert ist. Im Rahmen des Kyoto-Protokolls haben sich allein die Industrieländer zu Emissionsreduktionen verpflichtet, wohingegen die Entwicklungs- und Schwellenländer keine derartigen Verpflichtungen eingehen mussten. Bei den Verhandlungen über ein Nachfolgeabkommen in Kopenhagen im Jahr 2009 waren aber die Entwicklungs- und Schwellenländer nicht bereit, dem guten Beispiel der Industrieländer zu folgen und ihrerseits auch einen Beitrag zum Klimaschutz zu leisten. Deshalb - und auch weil einige Industrieländer nicht länger gewillt waren, die Vorreiterrolle zu spielen - scheiterten diese Verhandlungen und damit die Bemühungen um eine international koordinierte Klimapolitik (Nordhaus 2019, S. 2007-2008).

Es ist sogar möglich, dass andere Länder von den Klimaschutzmaßnahmen eines Landes in zweifacher Weise profitieren. Das ist dann der Fall, wenn emissionsintensive Industrien aus dem klimapolitisch ambitionierteren Land in Drittländer aufgrund deren klimapolitischer Standortvorteile abwandern („,carbon leakage“). Außerdem kann es sein, dass der in einem klimapolitisch aktiven Land erreichte Rückgang der Nachfrage nach fossilen Brennstoffen zu einem Sinken der Preise derselben und in der Folge zu einem Anstieg der Nachfrage in anderen Ländern führt (,green paradox"). In diesen Fällen werden die einseitigen Maßnahmen des einen Landes nicht nur nicht zu einer aktiveren Klimapolitik in anderen Ländern, sondern sogar zu höheren Treibhausgasemissionen in diesen führen - und dann wäre der Nettoeffekt von nationalen Emissionszielen sogar noch geringer, als es dem Anteil dieses Landes an 
Abb. 2 Länderanteile an globalen $\mathrm{CO}_{2}$-Emissionen 2019. (Quelle: eigene Darstellung nach Daten aus Crippa 2020, S. 19)

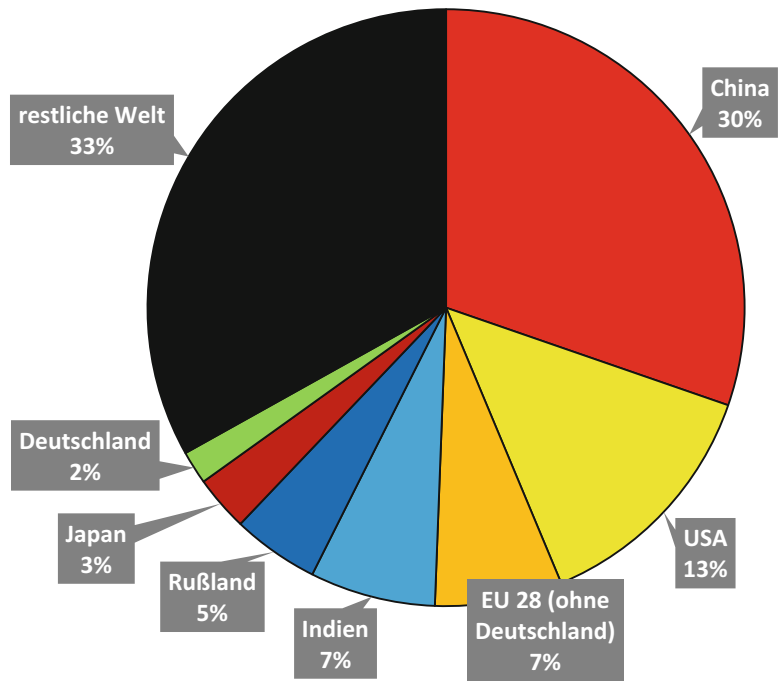

den Emissionen entspricht (Edenhofer et al. 2020, S. 13; SVR 2019, S. 26; Sinn 2008).

Es bleibt festzuhalten, dass einseitige Klimaschutzmaßnahmen einzelner Länder weder sinnvoll noch zielführend sind: Sie sind es nicht in klimapolitischer Hinsicht, weil sie keinen signifikanten Beitrag zur weltweiten Emissionsreduzierung leisten; und sie sind es aber auch nicht in wirtschaftspolitischer Hinsicht, weil sie enorme Kosten und Wettbewerbsverzerrungen verursachen.

Wenn man aber unterstellt, dass die Politik aus nicht-, außer- oder überökonomischen Gründen trotzdem europäische bzw. nationale Emissionsziele verfolgt, kann die Ökonomie zumindest dazu beitragen, dass solche Instrumente eingesetzt werden, die die angestrebten Emissionsziele mit den geringstmöglichen Kosten erreichen.

\section{Die Instrumente der Klimapolitik}

Da das Klimaproblem als globales Problem auch global gelöst werden muss, also durch ein international koordiniertes Vorgehen, stellt sich zunächst die Frage, wie ein solches zustande gebracht werden kann (Teil 4.1). Nur wenn dies gelingt, steht die Entscheidung an, welche Instrumente im Rahmen einer international koordinierten Klimapolitik einzusetzen sind (Teil 4.2). Neben der Reduktion der Treibhausgasemissionen dürfen Maßnahmen zur Anpassung an den Klimawandel nicht vernachlässigt werden, da sich dieser allenfalls verlangsamen oder aufhalten, nicht aber kurz- oder mittelfristig umkehren lassen wird (Teil 4.3). Schließlich erscheint es angesichts der Tragweite des Klimaproblems auch angebracht, nicht nur den Einsatz vorhandener Verfahren zu optimieren, sondern auch über die Förderung der Entwicklung neuer Technologien nachzudenken (Teil 4.4.). 
Abb. 3 Emissionsreduktion bei Kooperation und bei Nichtkooperation

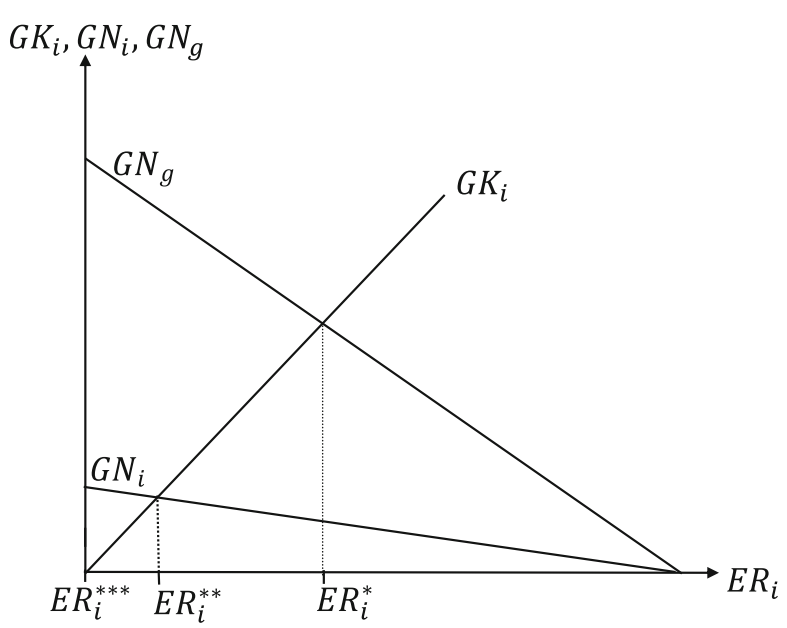

Da $\mathrm{CO}_{2}$ der Hauptverursacher des Treibhauseffekts ist, konzentrieren wir uns im Folgenden auf dieses Gas. Unsere Ausführungen lassen sich unschwer - mutatis mutandis - auf Treibhausgase im Allgemeinen übertragen.

\subsection{Internationale Umweltabkommen und Klimaschutz}

Der Schutz des Klimas stellt ein globales, nicht-ausschließbares öffentliches Gut dar. Eine ökonomisch rationale Klimapolitik muss deshalb eine globale, also international koordinierte Klimapolitik sein (vgl. Teil 3). Das entscheidende Hindernis auf dem Weg zu einer solchen besteht in dem Auseinanderfallen von globaler Problemnatur und nationaler Problemlösungskompetenz. Denn es gibt keine Weltregierung oder keine globale Umweltbehörde, die die Kompetenz hätte, dieses Problem mittels geeigneter klimapolitischer Maßnahmen zu lösen. Diese sind und bleiben vielmehr Sache der Nationalstaaten (oder von Staatengemeinschaften wie der EU). Eine globale Klimapolitik kann also nur durch freiwillige Kooperation im Rahmen internationaler Umweltverträge zustande kommen. Tragischer Weise ist aber die internationale Zusammenarbeit gerade in den Fällen am schwierigsten, in denen sie am notwendigsten wäre - nämlich bei globalen Problemen wie dem Klimaproblem. In diesem Fall ist nämlich der Konflikt zwischen individueller und kollektiver Rationalität besonders stark ausgeprägt. Die Kosten des Klimaschutzes müssen von jedem Land, das entsprechende Maßnahmen ergreift, vollständig getragen werden, während der Nutzen dieser Maßnahmen der ganzen Welt, also dem betreffenden Land nur zu einem kleinen Teil zu Gute kommt. Jedes einzelne Land wird also nur insoweit Klimapolitik betreiben, als diese ihm selbst nutzt; die positiven externen Effekte der eigenen Politik auf die anderen Länder werden vernachlässigt.

Diese Problematik wird in Abb. 3 veranschaulicht: $G K_{i}$ bezeichnet die Grenzkosten der Emissionsreduktion $\left(E R_{i}\right)$ des Landes $i, G N_{i}$ den Grenznutzen dieser Reduktion für das Land $i$ und $G N_{g}$ den globalen Grenznutzen der Emissionsreduktion. Global effizient wäre das Reduktionsniveau $E R_{i}^{*}$, bei dem $G K_{i}$ und $G N_{\mathrm{g}}$ gleich sind. Falls das Land $i$ unabhängig vom Rest der Welt seine Emissionen redu- 
ziert und die Konsequenzen seiner Politik auf die anderen Länder ignoriert, wird es $G K_{i}$ und $G N_{i}$ zum Ausgleich bringen, also nur das (aus globaler Sicht) ineffizient niedrige Reduktionsniveau $E R_{i}^{* *}$ realisieren.

Verhält sich das Land strategisch und hofft darauf, dass andere Länder ihre Emissionen reduzieren und es dann von deren Anstrengungen profitieren kann, ohne dass ihm selbst Kosten entstehen, wird es überhaupt keine Klimapolitik betreiben $\left(E R_{i}^{* * *}\right.$ in Abb. 3). Wenn jedes Land eine solche „Trittbrettfahrer“-Strategie verfolgen würde, dann gäbe es natürlich überhaupt keine Emissionsreduktion. In der Realität dürfte es aber ohnehin in den allermeisten Fällen kaum einen Unterschied zwischen der Trittbrettfahrer-Strategie und dem Fall der unkoordinierten Emissionsreduktion geben. Denn je größer die Zahl der Länder ist, also je geringer das Ausmaß ist, in dem ein einzelnes Land das Klima beeinflussen kann, desto flacher wird die $G N_{i}$ Kurve verlaufen und desto mehr wird sich $E R_{i}^{* *}$ an $E R_{i}^{* * *}$ annähern. Eine nennenswerte unkoordinierte Emissionsreduktion wird deshalb nicht stattfinden; eine Ausnahme könnten allenfalls einige wenige, sehr große Länder bilden. In einer solchen Situation würden aber alle Länder Nachteile erleiden, da ja (annahmegemäß) eine Begrenzung des Temperaturanstiegs im Interesse aller ist.

Solche nicht-kooperativen Gleichgewichte sind nicht die einzig möglichen Lösungen. Spieltheoretische Überlegungen zeigen, dass eine freiwillige Kooperation durchaus zustande kommen kann - allerdings nur in einem sehr begrenzten Umfang (Barrett 1990, 1994). Unter den für das Klimaproblem charakteristischen Bedingungen (viele Verursacher, viele Geschädigte, hohe mögliche Schäden, hohe Vermeidungskosten) ist eine freiwillige Kooperation nur für einige wenige Länder individuell rational; für die meisten Länder ist die ,Trittbrettfahrer“-Strategie lohnender. Ein stabiles internationales Klimaabkommen würde von daher nur wenige Vertragsparteien haben - und wäre deshalb ineffektiv. Zur Überwindung dieses Dilemmas zwischen kollektiver und individueller Rationalität hat Nordhaus (2015) „Klimaclubs“ vorgeschlagen, die sich von ,normalen“ Umweltabkommen dadurch unterscheiden, dass Länder, die einem solchen Club nicht beitreten, sanktioniert werden - und zwar durch Strafzölle auf die Exporte dieser Länder in die Mitgliedsländer des Klimaclubs. Gründungsmitglieder eines solchen Clubs könnten die Länder sein, die auch im Rahmen konventioneller Umweltabkommen freiwillig miteinander kooperieren würden; denn schließlich setzt auch die Gründung eines Klimaclubs die freiwillige Kooperation zumindest der Gründungsmitglieder voraus. Die gewünschte Wirkung kann ein Klimaclub nur entfalten, wenn er die notwendige „kritische Mas-

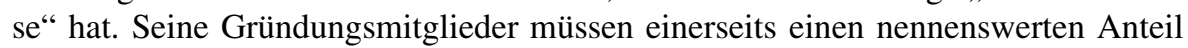
der globalen $\mathrm{CO}_{2}$-Emissionen auf sich vereinigen und andererseits müssen deren Märkte so bedeutend sein, dass eine Erschwerung des Marktzugangs durch Strafzölle tatsächlich einen Anreiz zum Clubbeitritt darstellen würde. ${ }^{2}$ Es erscheint sicher, dass ein Club, der die EU, das Vereinigte Königreich, die USA, Kanada, Australien,

\footnotetext{
2 Solche Klimazölle würden allerdings kaum WTO-konform sein, da sie nicht lediglich einen Ausgleich der den Unternehmen in den Mitgliedsländern durch klimapolitische Maßnahmen entstehenden Belastungen, also nicht nur die Herstellung von Wettbewerbsneutralität, bezwecken, sondern dezidiert der „Bestrafung“ von Nicht-Clubmitgliedern dienen würden. Inwieweit sie gemäß dem Motto „Might makes right“ dennoch durchsetzbar wären, ist freilich eine andere Frage.
} 
Japan, Russland, China und Indien umfassen würde, diese Bedingungen erfüllen und im Lauf der Zeit weitere Mitglieder anziehen würde. Er könnte so zur Keimzelle für eine weltweit koordinierte Klimapolitik darstellen. Weniger wahrscheinlich, aber immerhin möglich wäre es, dass ein solcher Club auch funktionieren würde, wenn zu ihm (zunächst) nur die wichtigsten ,,alten“ Industriestaaten gehörten, also Russland, China und Indien keine Mitglieder wären.

Mehr als zweifelhaft ist aber, ob das Pariser Klimaabkommen dazu geeignet ist, das Problem des Trittbrettfahrerverhaltens zu überwinden und eine global koordinierte Klimapolitik zu initiieren. Jedes Land hat sich nämlich unabhängig voneinander zu den Maßnahmen verpflichtet, die es für sinnvoll und im eigenen Interesse hält; es überrascht deshalb nicht, dass diese Maßnahmen nicht mit dem 1,5-Grad-Celsius-Ziel, zu dem man sich bekannt hat, vereinbar sind; denn auch bei Einhaltung aller Selbstverpflichtungen würde dieses Ziel nicht erreicht werden. Ganz abgesehen davon gibt es keinerlei Sanktionen bei Nichterreichung des Klimaziels oder bei Nichterfüllung der Selbstverpflichtungen - oder bei einem Ausstieg eines Landes aus dem Pariser Abkommen (Nordhaus 2019, S. 2007-2008). Letztlich wurde nur das bisherige unkoordinierte Nebeneinander nationaler Klimapolitiken institutionalisiert - und das Versagen der internationalen Staatengemeinschaft in der Klimafrage durch wohlklingende Zielvereinbarungen bemäntelt. „The world is therefore just where it stood in 1994, recognizing the dangers of climate change without effective policies to stop it“" (Nordhaus 2019, S. 2008).

\subsection{Kosteneffizienz durch einen einheitlichen $\mathrm{CO}_{2}$-Preis}

Anstrengungen zur Reduktion von $\mathrm{CO}_{2}$-Emissionen sind nur dann effektiv, können nur dann klimastabilisierend wirken, wenn sie koordiniert erfolgen - entweder, und das wäre der Idealfall, weltweit oder, in einem ersten Schritt, im Rahmen eines Klimaclubs. ${ }^{3}$ Welcher Instrumente sollte sich eine solche international koordinierte Klimapolitik bedienen? Aus umweltökonomischer Sicht ist ganz klar ein einheitlicher Preis für den Ausstoß von Treibhausgasen notwendig, der entweder direkt durch eine Emissionssteuer oder indirekt durch ein Emissionszertifikatssystem eingeführt werden könnte. Im ersten Fall würde der Steuersatz festgelegt und die Emissionsmenge würde sich gemäß den Reaktionen der Emittenten ergeben; im zweiten Fall würde die Zertifikats- bzw. Emissionsmenge bestimmt und der Zertifikatspreis würde sich durch Angebot und Nachfrage am Markt bilden (,cap and trade“). Entscheidend ist, dass in beiden Fällen einheitlich vorgegangen wird, d.h. dass alle Emittenten in allen Ländern in gleichem Maße belastet werden. Nur so können die Treibhausgasemissionen effektiv und effizient, d.h. zu den geringstmöglichen Kosten reduziert werden. Denn auf diese Weise würden die Kosten des Klimawandels den Verursachern angerechnet und diese hätten einen Anreiz, die Treibhausgasemissionen zu senken. Dabei würden die Emittenten ihre Emissionen umso stärker

\footnotetext{
3 Sollten einzelne Länder unkoordinierte (Netto-) Emissionsreduktionen durchführen (das Gleichgewicht $E_{i}^{* *}$ in Abb. 3), so gelten die folgenden Ausführungen auch für diese. Selbst wenn solche Maßnahmen (fast) keine Auswirkungen auf das Klima haben und deswegen eigentlich sinnlos sind, sollten sie zumindest kosteneffizient sein.
} 
reduzieren, je niedriger ihre Reduktionskosten sind, sodass insgesamt die Emissionsreduktion zu den geringstmöglichen Kosten erfolgen würde. Beide Instrumente haben ihre Vor- und ihre Nachteile, aber die Steuerlösung erscheint hauptsächlich aus zwei Gründen das bessere Instrument zu sein: Erstens haben bei ihr die Haushalte und Unternehmen Planungssicherheit, da sie bei Kenntnis des Steuersatzes auch genau wissen, wieviel sie die Emission von Treibhausgasen kosten wird und sie ihr Verhalten dementsprechend anpassen können. Zweitens wäre bei einer Steuerlösung die schrittweise Ausdehnung eines Klimaclubs auf die gesamte Staatengemeinschaft leichter möglich als bei einer Zertifikatslösung. Im Fall der Steuerlösung müssten neue Clubmitglieder lediglich den vom Klimaclub festgelegten $\mathrm{CO}_{2}$-Preis übernehmen und national durch die Erhebung entsprechender Steuern implementieren. Bei der Zertifikatslösung wären dagegen bei Aufnahme jedes neuen Mitglieds Verhandlungen über die Erweiterung des Emissionsvolumens und unter Umständen über die Aufteilung desselben auf alle Mitglieder erforderlich. ${ }^{4}$

Eine effiziente Klimapolitik kann sich aber nicht auf die Reduktion der $\mathrm{CO}_{2}$ Emissionen beschränken, sondern muss auch Maßnahmen zur Entfernung von $\mathrm{CO}_{2}$ aus der Atmosphäre umfassen (vgl. dazu z. B. Rickels et al. 2019). Für die Klimaentwicklung entscheidend ist der $\mathrm{CO}_{2}$-Gehalt der Atmosphäre, der wiederum nicht von den Bruttoemissionen abhängt, sondern von den Nettoemissionen, also dem Saldo von $\mathrm{CO}_{2}$-Emissionen und der Menge von $\mathrm{CO}_{2}$, die wieder aus der Atmosphäre entfernt wird - und zwar entweder auf natürlichem Wege (hauptsächlich durch $\mathrm{CO}_{2^{-}}$ Absorption der Ozeane) oder mittels der negativen Emissionstechnologien. Zu diesen gehören biologische Verfahren (z.B. Aufforstung oder Renaturierung von Mooren) oder chemische Verfahren (z. B. mineralische Karbonatbildung oder marines Alkalinitätsmanagement). Es wäre ineffizient, zu hohen Kosten eine bestimmte Menge an $\mathrm{CO}_{2}$-Emissionen zu vermeiden, wenn man zu geringeren Kosten dieselbe Menge an $\mathrm{CO}_{2}$ aus der Atmosphäre mit Hilfe der erwähnten negativen Emissionstechnologien entfernen könnte. Unter Einbezug dieser Technologien könnte eine insgesamt kosteneffiziente Klimapolitik dann realisiert werden, wenn nicht nur ein einheitlicher Steuersatz auf alle $\mathrm{CO}_{2}$-Emissionen angewandt werden würde, sondern wenn gleichzeitig eine einheitliche Subvention in der diesem Steuersatz entsprechenden Höhe für alle $\mathrm{CO}_{2}$-Absorptionen gewährt werden würde (vgl. zu diesem Vorschlag Söllner 1993). Genauso wie der einheitliche Steuersatz dafür sorgen würde, dass die Emissionen dort vermieden werden, wo die Vermeidungskosten am geringsten sind, würde der einheitliche Subventionssatz sicherstellen, dass diejenigen negativen Emissionstechnologien zum Einsatz kommen, die am kostengünstigsten sind. Außerdem wäre durch die Gleichheit von Subventions- und Steuersatz gewährleistet, dass Reduktion und Absorption von $\mathrm{CO}_{2}$ im effizienten Verhältnis durchgeführt werden. $\mathrm{Ob}$ in diesem System ein Einnahmenüberschuss erzielt werden würde oder nicht, würde von den Verhältnissen der Elastizitäten der Reduktions- und der Absorptionskosten abhängen. Dies gilt zumindest für den Übergang bis zu einem globalen

\footnotetext{
${ }^{4}$ Bezieht man politische und administrative Erwägungen mit ein, kann man beim Instrumentenvergleich zu einem anderen Ergebnis kommen. So existiert in Europa mit dem EU-ETS schon seit über einem Jahrzehnt ein funktionierendes und erfolgreiches Zertifikatssystem, mit dessen Mechanismen alle Beteiligten inzwischen viele Erfahrungen gesammelt haben (vgl. Teil 5).
} 
Klimagleichgewicht, welches dadurch gekennzeichnet ist, dass die globale Nettoemission gleich null und folglich die $\mathrm{CO}_{2}$-Konzentration in der Atmosphäre konstant ist. Darin muss das Fernziel jeder Klimapolitik bestehen, unabhängig davon, wann und bei welcher $\mathrm{CO}_{2}$-Konzentration ein solches Gleichgewicht erreicht werden soll. Aufgrund der natürlichen $\mathrm{CO}_{2}$-Absorption würde eine Nettoemission von null bei einem einheitlichen Steuer- und Subventionssatz einen Einnahmenüberschuss implizieren: Die Nettoemission ist dann null, wenn die Bruttoemission gleich der Summe aus natürlicher und ,technologischer" Absorption ist; dementsprechend wären die Steuereinnahmen höher als die Subventionsausgaben, da ja keine Subventionen für die natürliche Absorption gezahlt werden bzw. dieselben nur einen kalkulatorischen Posten darstellen würden. In Höhe dieser fiktiven Subventionen würde sich deshalb ein Einnahmenüberschuss ergeben, der z. B. für Transfers an Entwicklungsländer verwendet werden könnte, um diese bei der Anpassung an den Klimawandel zu unterstützen.

\subsection{Kampf gegen oder Anpassung an den Klimawandel?}

So wichtig wie ein international koordiniertes Vorgehen bei der Reduktion der Nettoemissionen von $\mathrm{CO}_{2}$ auch ist, so kann sich die Klimapolitik doch nicht darauf beschränken. Der Klimawandel hat schon begonnen und er wird auch dann weitergehen, wenn es gelingen würde, die Nettoemissionen effektiv zu reduzieren. Selbst wenn diese auf null gesenkt werden könnten, würde sich das dann sich einstellende Klimagleichgewicht deutlich von den klimatischen Bedingungen vor Beginn des Klimawandels unterscheiden. Eine Rückkehr zum Ex-ante-Gleichgewicht würde aufgrund der langen Verweildauer der Treibhausgase in der Atmosphäre Jahrhunderte dauern, wenn sie überhaupt möglich wäre. ${ }^{5}$ Von daher ist auch eine Anpassung an die Folgen des Klimawandels notwendig. ${ }^{6}$ Maßnahmen wie die Verstärkung der Deiche an der Nordsee, die Verbesserung des Hochwasserschutzes an Flussläufen, der Umbau der Wälder oder die Installation von Klimaanlagen in Krankenhäusern und Pflegeheimen müssen ergriffen werden. Eine internationale Koordination der Adaptionsmaßnahmen ist weder erforderlich noch sinnvoll: Erforderlich ist sie nicht, weil sowohl Kosten als auch Nutzen lokal bzw. national anfallen und keine grenzüberschreitenden positiven externen Effekte existieren; Trittbrettfahrerverhalten kann es also nicht geben. Sinnvoll ist die Koordination deswegen nicht, weil die Folgen des Klimawandels von Land zu Land unterschiedlich sind; auch ist es nicht ausgeschlossen, dass einige Länder vom Klimawandel profitieren können.

Und wenn es nicht gelingt, eine international koordinierte Nettoemissionsreduktion herbeizuführen? In diesem Fall müssen die nationalen Anstrengungen allein der Adaption gelten. Unkoordinierte Reduktionsbemühungen einzelner Länder wären, wie wir gezeigt haben, ineffektiv und würden lediglich eine Verschwendung von Ressourcen darstellen. Konkret bedeutet dies für Deutschland, dass in der gegenwär-

\footnotetext{
5 Ein Einsatz von negativen Emissionstechnologien in einem Umfang, der die Erhöhung der $\mathrm{CO}_{2}-\mathrm{Kon}_{-}$ zentration in der Atmosphäre seit Beginn der Industrialisierung rückgängig machen würde, erscheint beim heutigen Stand der Technik utopisch.

6 Vgl. dazu z. B. Lomborg (2020, Kap. 13), Neubäumer (2019, S. 798), SVR (2019, S. 15-16).
} 
tigen Situation, die durch das ineffektive und ineffiziente Pariser Klimaabkommen gekennzeichnet ist, Reduktionsanstrengungen nur bis zu dem Punkt unternommen werden sollten, an dem der nationale Grenznutzen den nationalen Grenzkosten entspricht (Situation $E R_{i}^{* *}$ in Abb. 3). Im Fall Deutschlands als eines (nach globalem Maßstab) „kleinen“ Emittenten würde dies praktisch eine Emissionsreduktion von null implizieren.

Die deutsche Klimapolitik sollte unter den aktuellen Bedingungen also keine Reduktionsmaßnahmen implementieren, sondern sich auf die Bewältigung der Folgen des Klimawandels konzentrieren - und gleichzeitig durch Verhandlungen auf internationaler Ebene versuchen, diese Bedingungen dergestalt zu ändern, dass eine effektive und effiziente Klimapolitik möglich wird, in deren Rahmen nationale Emissionsreduktionen erst sinnvoll sind.

\subsection{Die Förderung von Forschung und Entwicklung}

Bei einem langfristigen Problem wie dem des Klimawandels ist es wenig sinnvoll, nur eine statische Betrachtung anzustellen, also insbesondere von gegebenen Technologien und konstanten Kosten- bzw. Produktionsfunktionen auszugehen. Vielmehr ist im Zeitablauf mit an Sicherheit grenzender Wahrscheinlichkeit mit technischem Fortschritt und der Entwicklung neuer Technologien zu rechnen. Dadurch können die Kosten sowohl der $\mathrm{CO}_{2}$-Emissionsreduktion als auch der Entnahme von $\mathrm{CO}_{2}$ aus der Atmosphäre gesenkt werden, sodass ein gegebenes klimapolitisches Ziel mit geringeren Kosten oder bei gegebenen Kosten ein ehrgeizigeres Ziel erreicht werden kann. Bekanntlich werden - anders als bei ordnungsrechtlichen Eingriffen - schon durch die Bepreisung der $\mathrm{CO}_{2}$-Emissionen und die Subventionierung der $\mathrm{CO}_{2}-\mathrm{Ab}$ sorption Anreize geschaffen, die Reduktions- bzw. Absorptionstechnologien weiter zu entwickeln. Man spricht in diesem Zusammenhang auch von der ,dynamischen Effizienz“ dieser Instrumente - im Unterschied zur (statischen) Kosteneffizienz.

Darüber hinaus kann es sinnvoll sein, staatlicherseits Forschung und Entwicklung von Technologien in den Fällen zu fördern, in denen die Entwicklungskosten sehr hoch oder die Erfolgswahrscheinlichkeit sehr klein und die positiven externen Effekte eines technologischen Durchbruchs sehr hoch sind. Als Beispiel wäre hier vor allem die Entwicklung der Kernfusionstechnologie zu nennen.

Kaum zu rechtfertigen ist dagegen die staatliche „Privilegierung bestimmter Technologien im Wettbewerb durch Einschränkung der Wahlfreiheit von Marktpartnern, die gesetzlich zu betrieblich unwirtschaftlichen Entscheidungen gezwungen werden“ (Wissenschaftlicher Beirat beim BMWA 2004, S. 15). Dies ist in Deutschland gegenwärtig vor allem bei der Förderung der Elektromobilität und der erneuerbaren Energien der Fall. Die staatlichen Eingriffe in diesen Bereichen stellen eine Anmaßung von Wissen à la Hayek dar, da der Wettbewerb bewusst zugunsten bestimmter Technologien verzerrt wird, bei denen ganz und gar nicht sicher ist, ob sie tatsächlich den effizientesten Weg darstellen, $\mathrm{CO}_{2} \mathrm{zu}$ reduzieren. Im Gegenteil, es ist offensichtlich, dass die Förderung dieser Technologien extrem ineffizient ist, sodass dieselbe zwar politökonomisch erklärt, aber nicht umweltpolitisch gerechtfertigt werden kann (vgl. Teil 6). Um beim Beispiel der Elektromobilität zu bleiben: Es gibt zwar gute Gründe, die Entwicklung neuer Batterietechnologien oder verbes- 
serter Brennstoffzellen zu fördern, nicht aber, die Entscheidungen der Nachfrager massiv zugunsten batterieelektrischer Fahrzeuge zu beeinflussen.

\section{Die Ineffizienzen der deutschen und der europäischen Klimapolitik}

Entspricht die in Deutschland und in Europa aktuell betriebene Klimapolitik den Anforderungen an eine ökonomisch rationale Klimapolitik? Dem ist mitnichten so. Man erkennt unschwer, dass die ökonomische Rationalität in der Klimapolitik praktisch keine Rolle spielt - und zwar sowohl auf deutscher als auch auf europäischer Ebene.

\subsection{Kosteneffizienz und deutsche Klimapolitik}

Da wir in einer Welt der Knappheit leben, sollte es auch in der Klimapolitik das zentrale Ziel sein, die volkswirtschaftlichen Kosten des Klimaschutzes möglichst gering zu halten. Dies ist dann gewährleistet, wenn die Reduktion der Treibhausgasemissionen kosteneffizient vorgenommen wird, wenn also die ,jeweils nächste Einheit Treibhausgasemissionen dort eingespart wird, wo dies nach dem aktuellen Stand der (technischen) Gegebenheiten am günstigsten möglich ist“" (SVR 2019, S. 11). Da niemand schon im Voraus weiß, was die langfristig kostengünstigste Option oder Technologie ist, ist es daher unumgänglich, einen gesellschaftlichen Suchprozess nach den kostengünstigsten Vermeidungsoptionen in Gang zu setzten. Dieser Suchprozess ist umso effizienter, je mehr Akteure aus den verschiedenen Ländern und Sektoren beteiligt sind, was einen einheitlichen, alle volkswirtschaftlichen Sektoren umfassenden Ansatz erfordert (SVR 2019, S. 11-12).

Im Gegensatz dazu ist die Klimapolitik in Deutschland - nach Meinung des Sachverständigenrats (2019) und des Wissenschaftlichen Beirats beim BMWi (2019) kleinteilig, ineffizient und leistet (fast) keinen Beitrag zur klimapolitisch erwünschten Verringerung der Treibhausgasemissionen. Dies gilt vor allem für das im Jahr 2000 eingeführte Erneuerbare-Energien-Gesetz (EEG), das den Ausbau erneuerbarer Energien bei der Stromerzeugung fördert und zu diesem Zweck feste Einspeisevergütungen für erneuerbare Energieanlagen garantiert und für diese eine Anschlusspflicht und den Einspeisevorrang vorsieht. Kernelement ist die technologiespezifische umlagefinanzierte Förderung über eine garantierte Laufzeit von 20 Jahren (die sogenannte „EEG-Umlage“). Der Wissenschaftliche Beirat beim BMWA (2004, S. 6) hatte schon im Jahr 2004 darauf hingewiesen, dass eine direkte Förderung erneuerbarer Energien (durch das EEG) innerhalb des übergreifenden EU-Emissionshandelssystem keinen Beitrag zur Verringerung der Treibhausgasemissionen erbringt, sondern nur zu einer Verlagerung in andere Länder führt. Im Übrigen ist fraglich, ob (allein oder hauptsächlich) mit regenerativen Energien und - auf absehbare Zeit - ohne ausreichende Stromspeicher die Versorgungssicherheit für den Industriestandort Deutschland in Zukunft gewährleisten werden kann (Farnung 2021). Der Kohleausstiegsbeschluss, die massive Förderung der Elektromobilität und das „Klimapaket 2030“ sind weitere Weichenstellungen der deutschen Klimapolitik, die diese Linie fortführen. Hinzu kommt, dass all diese nationalstaatlichen energie- und klimapolitischen Entschei- 
dungen Deutschlands ohne enge Abstimmung mit seinen Nachbarn bzw. mit der EU erfolgt sind, was angesichts der Tatsache, dass es sich beim Klimaproblem um das grenzüberschreitende Umweltproblem par excellence handelt, doch mehr als nur ein wenig erstaunt.

Angesichts eines solchen Vorgehens überraschen enorme Ineffizienzen bei der Reduktion von $\mathrm{CO}_{2}$-Emissionen nicht. Bei einem Vergleich der durchschnittlichen errechneten $\mathrm{CO}_{2}$-Vermeidungskosten (in Euro/t vermiedener $\mathrm{CO}_{2}$-Emissionen) kommt man je nach den getroffenen Annahmen zwar zu unterschiedlichen Aussagen, deren Grundtenor jedoch eindeutig ist: Eine Studie der Initiative $\mathrm{CO}_{2}$-Abgabe beziffert die spezifischen $\mathrm{CO}_{2}$-Vermeidungskosten der Windenergie onshore auf ca. $1900 €$ und die der Photovoltaik auf ca. $1874 €\left(\mathrm{CO}_{2}\right.$-Abgabe 2019, S. 10). Für den Bereich der Elektromobilität sind die spezifischen Vermeidungskosten - wie Weimann (2020, S. 894) jüngst dargelegt hat - auf 1100 bis $1200 € / t$ zu veranschlagen. Für den Kohleausstieg, dessen Gesamtkosten mindestens $100 \mathrm{Mrd}$. $€$ betragen werden, lassen sich die spezifischen Vermeidungskosten noch nicht genau beziffern, aber es deutet viel darauf hin, dass die Abschaltung moderner Kohlekraftwerke nicht die kostengünstigste Möglichkeit zur Reduktion von $\mathrm{CO}_{2}$-Emissionen darstellt (BDI 2020; Weimann 2019). Wenn man diese hohen Vermeidungskosten dem derzeitigen Marktpreis im EU-ETS von ca. $40 €$ pro Tonne $\mathrm{CO}_{2}$-Emissionen gegenüberstellt, so ergibt sich ein Faktor von ca. 27 bis 48, um den die aufgeführten $\mathrm{CO}_{2}$-Vermeidungsmaßnahmen bzw. -technologien teurer sind als der EU-ETS-Preis. Es ist klar, dass wir mit diesen verschiedenen Maßnahmen bei weitem nicht den kosteneffizientesten Weg eingeschlagen haben und genau das Gegenteil einer volkswirtschaftlich effizienten Klimapolitik betreiben. Hinzukommt, dass diese allenfalls auf nationaler Ebene effektiv ist, also eine Reduktion der $\mathrm{CO}_{2}$-Emissionen bewirken kann. Auf europäische Ebene ist die deutsche Klimapolitik dagegen zum größten Teil ineffektiv. Insbesondere lässt sich, was die Stromerzeugung angeht, innerhalb des übergreifenden EU-ETS, keine Vermeidungswirkung erzielen - zumindest nicht ohne weitere Maßnahmen, die ihrerseits zusätzliche Kosten verursachen würden. ${ }^{7}$

\subsection{Der europäische Green Deal}

Im Dezember 2019 haben sich die Staats- und Regierungschefs der Mitgliedsstaaten der EU auf den von der EU-Kommission vorgeschlagenen „Green Deal“ politisch geeinigt. ${ }^{8}$ Beim europäischen Green Deal handelt es sich laut EU-Kommission um eine Wachstumsstrategie, mit der die EU zu einer fairen und wohlhabenden Gesellschaft mit einer modernen, ressourceneffizienten und wettbewerbsfähigen Wirtschaft werden soll. In der Folge hat die Kommission einen Vorschlag für ein ,Europäisches Klimagesetz“ vorgelegt, in dem das Ziel der Klima- bzw. Treibhausgasneutralität der EU bis zum Jahr 2050 rechtsverbindlich festgeschrieben wird (Europäische Kommission 2019). Dazu will die EU alle Sektoren - Energiewirtschaft, Gebäude, Industrie,

\footnotetext{
${ }^{7}$ Diese Ineffektivität, die auf das Ziel der Reduktion der $\mathrm{CO}_{2}$-Emissionen bezogen ist, muss von der Ineffektivität jeder nur nationalen bzw. nur europäischen Politik mit Bezug auf das Ziel der Klimastabilisierung unterschieden werden (vgl. Teil 3).

8 Vgl. zum Folgenden Söllner (2020).
} 
Verkehr und Landwirtschaft - einbinden und Maßnahmenpakete zur Emissionsminderung und Ressourcenschonung implementieren. Dies wird mit weitreichenden Konsequenzen für die Wirtschaft und für das Leben der Bürger in der EU verbunden sein, die aber erst in den nächsten Monaten und Jahren transparent werden.

Doch ist der Green Deal überhaupt klimapolitisch sinnvoll und wirtschaftspolitisch tragbar? Wir haben schon dargelegt, dass es angesichts der globalen Natur des Klimaproblems wenig sinnvoll ist, dass einzelne Länder als Vorreiter vorangehen. Die EU-Kommission hat es dennoch getan und sich die Empfehlungen des IPCC zu eigen gemacht und selbst ambitionierte Ziele formuliert. Was im Pariser Abkommen noch eine weitgehend politische Deklaration der 195 Unterzeichnerstaaten war, hat die EU nun rechtsverbindlich festgelegt: Die Nettoemission von Treibhausgasen soll bis zum Jahr 2050 auf null sinken und als Zwischenziel wird für das Jahr 2030 eine Reduktion um mindestens 55\% (gegenüber 1990) angestrebt (Von der Leyen 2020). Dies entspricht einer tatsächlichen Reduktion um bis zu $48 \%$ gegenüber dem Jahr 2010, was das herausfordernde Ambitionsniveau der EU widerspiegelt. Da der Anteil der EU an den weltweiten Treibhausgasemissionen aber lediglich ca. 10\% beträgt (2017), wird die EU auch bei vollständiger Erreichung ihrer eigenen Klimaziele keine spürbare Reduktion der Gesamtemissionen und damit keine Stabilisierung des Klimas weltweit bewirken können.

Gleichzeitig ist zu erwarten, dass die anderen Länder zunächst einmal abwarten und selbst keine teure Klimapolitik betreiben. Und es ist sogar möglich, wie in Teil 3 gezeigt wurde, dass die einseitigen Maßnahmen der EU nicht nur nicht zu einer aktiveren Klimapolitik in anderen Ländern, sondern sogar zu höheren Treibhausgasemissionen in diesen führen - und dann wäre der Nettoeffekt des Green Deal auf die globalen Emissionen sogar noch geringer, als es dem bescheidenen Anteil der EU an diesen Emissionen entspricht. Es bleibt festzuhalten, dass einseitige Klimaschutzmaßnahmen wie der europäische Green Deal weder in klimapolitischer noch in wirtschaftspolitischer Hinsicht sinnvoll sind.

Um die ambitionierten Klimaziele zu erreichen, muss nach Ansicht der EUKommission eine erhebliche „Investitionslücke“ geschlossen werden, was zusätzliche private und öffentliche Investitionen in Höhe von 260 Mrd. $€$ pro Jahr bis 2030 notwendig macht (Europäische Kommission 2019, S. 18-21). Da die Vermeidungskosten tendenziell sicher nicht sinken werden, werden sogar noch weitaus höhere Summen bis zum Jahr 2050 jährlich erforderlich sein, um das ambitionierte Klimaziel der EU zu erreichen. Bis zum Jahr 2050 dürften sich die privaten und öffentlichen Investitionen daher auf mindestens 7800 Mrd. $€$ belaufen. Die insgesamt anfallenden Kosten, zu denen beispielsweise auch die Belastung von Industrie und Haushalten durch höhere Energiepreise gehört, dürften noch wesentlich höher sein. Modellrechnungen zeigen, dass nur für Deutschland eine Reduktion der Treibhausgasemissionen um $95 \%$ (bezogen auf 1990) Kosten von über 1,5 bis 2,3 Billionen Euro verursachen würde - und dies unter der Voraussetzung kosteneffizienter Reduktionsmaßnahmen (vgl. BDI 2018, S. 10-18). Berücksichtigt man, dass eine Reduktion der Emission aller Treibhausgase um fast $100 \%$ in der gesamten EU angestrebt wird und dass die Reduktionskosten pro Einheit Treibhausgasemission stark ansteigen, je mehr die Emissionen schon reduziert wurden, dann dürften die 
Gesamtkosten schätzungsweise in der Größenordnung von 15 bis 20 Billionen Euro liegen. ${ }^{9}$ Dies ist ein stolzer Preis für einen minimalen Klimaeffekt.

Wenn man aber akzeptiert, dass aus nichtökonomischen Gründen die Emissionsziele des europäischen Green Deal gesetzt sind, so kann gefragt werden, mit welchen Instrumenten man diese Ziele erstens erreichen (Effektivität) und zweitens die dabei entstehenden Kosten minimieren kann (Effizienz). Dafür sind prinzipiell sowohl eine einheitliche Treibhausgassteuer als auch ein einheitliches Treibhausgas-Zertifikatssystem geeignet (vgl. Teil 4.2). Beides existiert in der EU nicht. Stattdessen gibt es ein Nebeneinander von einerseits EU-weiten klimapolitischen Maßnahmen und andererseits unterschiedlichen nationalen Regelungen in den einzelnen Mitgliedsstaaten. Daran wird sich auch durch den Green Deal nichts ändern. Der „Maßnahmenkatalog“, den die EU-Kommission als Anhang zum europäischen Green Deal vorgelegt hat, enthält zwar Ziele, Absichtserklärungen und Appelle - aber eben kaum konkrete Maßnahmen. In Artikel 5 und 6 der Verordnung ist vielmehr die Rede von der „Bewertung der Fortschritte und Maßnahmen der Union“ und der „Bewertung der nationalen Maßnahmen“. Man muss also davon ausgehen, dass es bei dem bisherigen Nebeneinander europäischer und nationaler Maßnahmen bleiben wird, was sowohl die Effektivität als auch die Effizienz der Klimapolitik der EU beeinträchtigen wird.

Klimapolitische Instrumente können entweder effektiv sein, d.h. zur Reduktion der Treibhausgasemissionen beitragen - oder eben nicht. Im ersten Fall kann man außerdem überlegen, ob das Instrument auch effizient ist, d. h. keine unnötigen Kosten verursacht - oder eben nicht. Im zweiten Fall liegt immer Ineffizienz vor, denn wenn ein Instrument nichts zur Zielerreichung beiträgt, dann ist es zwangsläufig ineffizient, gleichgültig, wie hoch die Kosten sind. Wir werden zeigen, dass gerade das Nebeneinander europäischer und nationaler Maßnahmen auf jeden Fall und zwangsläufig Kostenineffizienzen verursacht, da jedes Abweichen von EU-weiten einheitlichen Maßnahmen vermeidbare Kosten nach sich zieht.

Auf EU-Ebene gibt es mit dem europäischen Emissionshandelssystem (EU-ETS) seit 2005 ein Instrument, das effektiv und effizient zugleich ist: Es gilt für die Energiewirtschaft, die verarbeitende Industrie und auch (seit 2012) für den europäischen Flugverkehr und umfasst ca. $45 \%$ der in Europa entstehenden $\mathrm{CO}_{2}$-Emissionen. ${ }^{10}$ Es liegt im Wesen eines solchen Systems, dass die politisch vorgegebene Emissionsobergrenze (,,cap“) nicht überschritten wird, dass aber der Preis für Zertifikate am Markt (,trade“) durchaus schwanken kann bzw. soll. Die bisherigen Emissionsreduktionen zeigen, dass dieses System in dem Sinne erfolgreich war, als dass es das ihm gesteckte Ziel (Verringerung der $\mathrm{CO}_{2}$-Emissionen der betreffenden Sektoren bis 2030 um $40 \%$ gegenüber 1990) zum Großteil schon erreicht hat und auf dem besten Wege ist, dieses vollständig zu erreichen - und zwar zu minimalen Kosten (Wei-

\footnotetext{
9 Da die Nettoemissionen auf null sinken sollen, müssen die Bruttoemissionen nicht um $100 \%$ reduziert werden, sondern „,nur“ soweit, dass sie den „negativen Emissionen“ (z. B. durch Aufforstung) entsprechen (vgl. Teil 4.2).

10 Seit 2013 werden auch fluorierte Gase und $\mathrm{N}_{2} \mathrm{O}$ einbezogen. Da der weit überwiegende Teil des Zertifikatshandels aber $\mathrm{CO}_{2}$ betrifft, vernachlässigen wir bei unserer Darstellung des EU-ETS die anderen Treibhausgase.
} 
mann 2019, S. 9). Anlass zu Kritik gibt nicht das System als solches, sondern die Tatsache, dass es bislang nicht konsequent genug angewendet wurde und deshalb sein Potenzial, die $\mathrm{CO}_{2}$-Emissionen effektiv und effizient $\mathrm{zu}$ reduzieren, nur zum Teil genutzt wurde.

Einerseits wird in verschiedenen Wirtschaftssektoren mit unterschiedlichen Instrumenten Klimapolitik betrieben: Beispielsweise gelten im Verkehrssektor Verbrauchs- bzw. $\mathrm{CO}_{2}$-Emissionsvorschriften für Kraftfahrzeuge („Flottengrenzwerte“) und, damit verbunden, ,Strafzahlungen“ für deren Hersteller. Da es beim Klimaproblem ja um die absolute Menge an Emissionen geht, macht es aber keinen Sinn, eine $\mathrm{CO}_{2}$-Bepreisung auf die relativen Emissionen $\left(\mathrm{CO}_{2}\right.$-Ausstoß/km) und nicht auf die absoluten Emissionen abzustellen. Wesentlich sinnvoller wäre es, das $\mathrm{CO}_{2}$-Zertifikatssystem auf den gesamten Verkehrssektor (und auch den Gebäudesektor) auszudehnen. Dann würden sich auch die verschiedenen Anreize auf nationaler Ebene zum Erwerb von Elektrofahrzeugen erübrigen, die - wie schon das EEG - eine technologie- und sektorspezifische, mithin also ineffiziente Subventionierung darstellen. Mit der Verteuerung von konventionellen Kraftstoffen würden stattdessen alle alternativen Antriebsformen attraktiver und das beste Konzept würde sich im Wettbewerb durchsetzen - sei es das Elektroauto, der Wasserstoffantrieb oder ein optimierter Verbrennungsmotor oder eine Mischung von allen. Ähnlich könnte man hinsichtlich der Vorgaben zur Wärmedämmung und zum Energieverbrauch von Gebäuden argumentieren: Diese könnten dadurch überflüssig werden, dass man das $\mathrm{CO}_{2}$-Zertifikatssystem auf alle fossilen Brennstoffe für die Wärmeerzeugung anwendet (also Heizöl und Erdgas). Und dann hätte auch die in Deutschland und den anderen EUMitgliedsstaaten auf fossile Brennstoffe erhobene Energiesteuer, die ja vor allem mit der Klimaschädlichkeit dieser Energieträger begründet wird, keine Daseinsberechtigung mehr - genauso wenig wie die als Teil des deutschen „Klimapakets 2030“ geplante $\mathrm{CO}_{2^{-}}$, ,Abgabe“ auf Benzin, Diesel, Heizöl und Erdgas. ${ }^{11}$

Andererseits werden auch in den schon jetzt unter das EU-ETS fallenden Sektoren zusätzliche Maßnahmen ergriffen (z.B. das Glühlampenverbot oder die Stromverbrauchsgrenzwerte für Staubsauger und andere Elektrogeräte). Diese zusätzlichen Restriktionen sind im Rahmen des übergreifenden EU-ETS nicht nur überflüssig, sondern verursachen auch unnötige Kosten und bewirken letztlich nur eine Verlagerung der $\mathrm{CO}_{2}$-Emissionen in andere Sektoren.

Es ist nicht zu erkennen, dass es im Zuge des ambitionierten und kostenintensiven Green Deal zu einer grundsätzlichen Reform der bisherigen ineffektiven, ineffizienten und teuren Klimapolitik kommen wird - ganz im Gegenteil. Deshalb muss man davon ausgehen, dass die Realisierung des Green Deals noch wesentlich teurer wird, als eigentlich notwendig wäre und die oben genannten Kostenschätzungen noch weit übertroffen werden - und dass angesichts eines Emissionsziels, welches auf die Entwicklung des weltweiten Klimas allenfalls einen vernachlässigbaren

\footnotetext{
11 De jure wird zwar ab 2021 ein nationales Emissionshandelssystem für die nicht vom EU-ETS erfassten Sektoren eingeführt; da aber gleichzeitig der Zertifikatspreis fixiert wurde (von $25 €$ pro Tonne $\mathrm{CO}_{2}$ im Jahr 2021 bis zu $55 €$ pro Tonne $\mathrm{CO}_{2}$ im Jahr 2025), handelt es sich de facto um eine $\mathrm{CO}_{2}-\mathrm{Steuer}-$ zumindest für den genannten Zeitraum (Bundesregierung 2019). Im Übrigen sei angemerkt, dass das Nebeneinander von EU-ETS und nationalen Emissionshandelssystem zwangsläufig zu Ineffizienzen führen wird (und zwar unabhängig davon, ob letzteres als De-facto-Steuer ausgestaltet ist oder nicht).
} 
Einfluss haben wird. Damit hat die EU-Kommission die Chance vertan, aus dem unkoordinierten Nebeneinander der vielfach unsystematischen nationalen Klimapolitiken der Mitgliedsländer durch den Green Deal eine einheitliche und stringente EU-weite Klimapolitik zu entwickeln.

Der Green Deal, so wie er jetzt konzipiert ist, wird ein weiteres Problem verursachen, das in der öffentlichen Diskussion bislang zu kurz gekommen ist (vgl. zum Folgenden z. B. Quick 2019). Wenn andere Länder dem Beispiel der EU nicht folgen, was - wie wir oben gezeigt haben - realistischer Weise zu erwarten ist, werden die in der EU ansässigen Unternehmen erhebliche Wettbewerbsnachteile durch höhere Energie- und Produktionskosten haben. Dies wird zu Produktionsverlagerungen oder zu Marktanteilsverlusten führen. Um dem entgegenzusteuern, schlägt die EU-Kommission ein $\mathrm{CO}_{2}$-Grenzausgleichssystem vor, mit dem die Belastungen durch den Green Deal mittels eines nicht näher spezifizierten Steuer- bzw. Zollmechanismus ausgeglichen werden sollen (Europäische Kommission 2019, S. 6). Dieses Vorhaben ist mit zahlreichen Schwierigkeiten und Unwägbarkeiten verbunden. Erstens ist es praktisch nicht möglich, den Kostenvorteil von Nicht-EU-Produzenten bzw. den Kostennachteil von EU-Produzenten durch den Green Deal auch nur halbwegs genau zu ermitteln, sodass mit groben Pauschalierungen zur Ermittlung der Ausgleichssätze gearbeitet werden müsste. Zweitens stellt sich die Frage, welche Nicht-EULänder davon betroffen werden sollen: Nur die Länder, die ihre Verpflichtungen aus dem Pariser Abkommen nicht erfüllen? Das kommt angesichts der oft sehr wenig ehrgeizigen und wachsweich formulierten Verpflichtungen eher nicht in Frage - und außerdem würde dies bei den nächsten Weltklimaverhandlungen wohl eher weniger ambitionierte Zielvorgaben vieler Länder bewirken. Also werden wahrscheinlich alle Länder gemeint sein, die sich nicht ähnlich ehrgeizige Ziele setzen und ähnlich rigorose Maßnahmen ergreifen wie die EU. Aber dies würde natürlich dem „Geist der Zusammenarbeit“ des Pariser Abkommens widersprechen. Drittens würde es keinen ,echten“ Ausgleich geben, denn dazu müssten nämlich nicht nur die Importe in die EU belastet, sondern auch die Exporte aus der EU entlastet werden, d.h. den Produzenten müssten die durch den Green Deal verursachten Zusatzkosten erstattet werden. Das ist aber klimapolitisch eindeutig nicht gewollt, sodass es bei einer einseitigen Belastung der Importe bleiben wird. Aus diesem Grund wäre, viertens, das Grenzausgleichssystem trotz gegenteiliger Beteuerungen der EU eben nicht WTOkonform - ganz abgesehen davon, dass ein Ausgleich ohnehin nur für Steuern erlaubt ist, die auf die Produkte selbst erhoben werden, nicht aber für die Kosten allgemeiner wirtschaftspolitischer Maßnahmen, seien sie nun klimapolitischer oder anderer Natur. Deshalb müsste die EU, fünftens, mit Gegenmaßnahmen ihrer Handelspartner rechnen. Angesichts des gegenwärtigen handelspolitischen Klimas würden diese höchstwahrscheinlich nicht auf eine Streitbeilegung im Rahmen der WTO setzen, sondern unverzüglich mit Vergeltungszöllen reagieren - was zu gravierenden Handelskonflikten und großen Wachstums- und Wohlstandseinbußen weltweit führen kann und die EU und insbesondere Deutschland mit seiner exportstarken Industrie treffen würde. Je ambitionierter und energischer die EU ihren Green Deal formuliert und durchsetzt, umso mehr bringt sie die Länder gegen sich auf, die selbst nicht ebenso ambitionierte Klimaziele verfolgen. 


\section{Zur politischen Ökonomie der Klimapolitik in Deutschland}

Aber warum ist die Klimapolitik in Deutschland so ineffizient und ineffektiv? Dieser Frage wollen wir im Folgenden nachgehen. Dabei beziehen wir uns zwar nur auf die deutsche Klimapolitik, doch dürften die beschriebenen Zusammenhänge und Mechanismen auch auf europäischer Ebene gelten. Die hier vorgestellten politökonomischen Erklärungsansätze werden hauptsächlich anhand des EEG erläutert dem Paradebeispiel für eine unsystematische, teure, ineffiziente und (zum Großteil) ineffektive Klimapolitik. ${ }^{12}$

\subsection{Die politische Attraktivität diskretionärer Eingriffe}

Effektiver Klimaschutz erfordert eine drastische Verringerung der globalen Treibhausgasemissionen und damit vor allem eine umfassende Transformation der Energieversorgungssysteme im Hinblick auf Treibhausgasneutralität. Da diese Umstrukturierungsprozesse enorm kostenintensiv sind, kommt gerade der Kosteneffizienz der Maßnahmen zentrale Bedeutung zu (Weimann und Timme 2019). Kosteneffizienz kann aber nur die marktwirtschaftliche Koordination bewirken, die mit Preissignalen dafür sorgt, dass Klimabelastungen dort vermieden werden, wo die Kosten der Vermeidung am niedrigsten sind. Die Monopolkommission (2009, S. 13) weist zu Recht darauf hin, dass ökonomische Ineffizienzen eine Volkswirtschaft belasten und damit auch den Handlungsspielraum für die Politik zur Erreichung von Klimazielen verringern.

Politische Entscheidungsträger haben ein starkes Interesse, nicht nur den Ordnungsrahmen für die Wirtschaft zu gestalten, sondern im wirtschaftlichen Geschehen bei Einzelentscheidungen selbst mitzuwirken. Politiker wollen unmittelbar an möglichst vielen konkreten (auch wirtschaftlichen) Entscheidungen so beteiligt sein, dass ihnen persönlich durch ihr Handeln ein positives Ergebnis zugerechnet wird. In vielen Fällen ist für die Politiker ihr ostentativ ,engagiertes“ Handeln, sprich: ihr Eingreifen in den Markt, entscheidend, nicht so sehr die tatsächlichen Auswirkungen ihres Verhaltens, die angesichts der Komplexität der wirtschaftlichen Zusammenhänge nicht klar zuordenbar sind. Politiker haben daher nur geringes Interesse, einen unpersönlichen und ergebnisoffenen Marktmechanismus zu etablieren und ihm bestimmte Entscheidungen anzuvertrauen (Schnellenbach 2020, S. 39). Es liegt also gar nicht in der Handlungslogik von Politikern, öffentlichkeitsbedeutsame Fragen in den ökonomischen Bereich auszulagern und dem Markt eine Chance zu geben. Die politische Entscheidungslogik ist vielmehr auf direkte, kurzfristig wirkende und zurechenbare Eingriffe der Politiker ausgerichtet. Dies gilt umso mehr, als eine diskretionäre Politik große Spielräume für die Gewährung von Sondervorteilen und die Optimierung der Wiederwahlchancen durch die gezielte Bevorzugung bzw. Benachteiligung bestimmter Wählergruppen mit sich bringt.

Wenn ein Marktergebnis aus politischer Sicht als unerwünscht interpretiert wird, wird es schnell als „Marktversagen“ abgewertet und eine politische Intervention gefordert. In vielen Fällen macht diese Intervention dann aber wiederum weitere

12 Vgl. zu den folgenden Ausführungen Pritzl (2020). 
politische Regulierungen erforderlich, sodass es $\mathrm{zu}$ einer Interventionsspirale mit immer weiteren legislativ-administrativen Eingriffen kommt. Nur in seltenen Fällen werden marktverzerrende staatliche Eingriffe zurückgenommen und bürokratische bzw. legislative Anforderungen abgebaut.

Das EEG ist ein gutes Beispiel dafür: In den vergangenen 20 Jahren hat sich ein ausdifferenziertes und selbst von Fachleuten kaum mehr handhabbares Regelwerk herausgebildet, das sich allein vom Umfang von einst 12 auf inzwischen 79 Paragraphen mehr als versechsfacht hat. Die Gesetzessammlung ,Energierecht“ aus dem Beck-Verlag bringt es mittlerweile, in der aktuellen 16. Auflage von 2020, auf über 2300 Seiten (gegenüber 512 Seiten der ersten Auflage 2000).

Diese kleinteilige Regelungsdichte ist nicht zuletzt das Resultat eines Verteilungskampfes um ökonomische Renten: Verschiedene durchsetzungsstarke Interessengruppen bemühen sich - weitgehend erfolgreich - um eine finanzielle Privilegierung (,Renteneinnahmen“), an der Wiederwahl orientierte politische Entscheidungsträger gewähren zahlreiche finanzielle Vorteile und Sondervorteile (z. B. über Ausnahmetatbestände im EEG) und die nur schwer organisierbare große Gruppe der Stromverbraucher trägt letztlich die Gesamtkosten (Cassel 2017, S. 46-48). Starke Lobbyakteure sind die Erneuerbare-Energien-Verbände (,Wind- und Solarlobby“), die Unternehmen der Energiewirtschaft und die einzelnen Bundesländer, die auf ihre natürlichen Standortvorteile (z. B. Wind im Norden, Sonne im Süden und Bioenergie in den agrarischen Flächenstaaten) verweisen. Auch die Klimaschutzbürokratie (zu der in Deutschland insbesondere das Umweltbundesamt gehört) entwickelt eine Eigendynamik und nutzt ihre politische Stellung zur Durchsetzung ihrer eigenen Präferenzen.

Die durch das EEG gewährten Renteneinahmen haben zu einer Herausbildung mächtiger Interessengruppen und zu einem Rent-Seeking-Spielfeld par excellence geführt (Ehler 2019). Die Lobbyakteure streben den weiteren mengenmäßigen Zubau von Erneuerbaren Energie-Anlagen an, der ihren Mitgliedern weitere lukrative EEG-Vergütungen für 20 Jahre garantiert, und wehren sich gegen eine Verringerung der Vergütungssätze bzw. Verschlechterung der Einnahmensituation. Die Kosten werden vor allem über die EEG-Umlage, die im Jahr 2020 auf den Rekordstand von $6,765 \mathrm{ct} / \mathrm{KWh}$ angestiegen ist, auf die Stromverbraucher abgewälzt. Die Gesamtkosten von EEG-Umlage, Stromsteuer und Netzentgelten belaufen sich laut Bundesrechnungshof (2018, S. 3) mittlerweile auf rd. 34 Mrd. $€$ pro Jahr, was durchschnittlich jeden Bundesbürger mit $415 €$ jährlich belastet. Und in diesem und im nächsten Jahr werden Haushaltsmittel in jeweils zweistelliger Milliardenhöhe verwendet, um die EEG-Umlage auf 6,5 bzw. 6,0 Cent zu deckeln. Das EEG führt zudem zu gesellschaftlich unerwünschten Umverteilungen zu Gunsten von Eigenheim- und Flächenbesitzern sowie Kapitaleignern, die zu Lasten der einkommensschwächeren Haushalte und Mieterhaushalte gehen (Techert et al. 2012).

Es kennzeichnet den enormen Einfluss der EEG-Lobbygruppen, dass sich, trotz der schon im Jahr 2004 vom Wissenschaftlichen Beirat beim BMWA (2004, S. 6-8) geäußerten Kritik, wenig an dieser kleinteiligen, ineffizienten und sozial unausgewogenen Grundstruktur der Förderung geändert hat. Je mehr die ökonomische Bedeutung dieser Branche aber zunimmt und je größer die Gruppe der erneuerbare Energien-Anlagen-Betreiber wird, desto weniger wird die Politik in Zukunft willig 
bzw. fähig sein, gegen deren Interessen zu handeln und die vom Sachverständigenrat geforderte Neuausrichtung der Energie- und Klimapolitik vorzunehmen. So manche Vertreter der EEG-Lobby nutzen die aktuelle Corona-Situation und plädieren für eine Weiterentwicklung der EEG-Förderung, indem sie ein Investitions- und Konjunkturprogramm geschickt mit einem beschleunigten Ausbau von Photovoltaik und Windkraft verknüpfen.

Das von der Bundesregierung Ende 2019 beschlossene „Klimapaket 2030“ ist ein weiteres Bespiel dafür, dass die Politiker kein Interesse an einer marktwirtschaftlich ausgerichteten Klimapolitik haben und - wie schon beim EEG - auf detaillierte Politiksteuerung über vielfältige Einzelmaßnahmen setzen (BMU 2019; Edenhofer et al. 2020). Der nationale $\mathrm{CO}_{2}$-Emissionshandel mit Fixpreissystem von 2021 bis 2025 entspricht in Wirklichkeit einer verkappten $\mathrm{CO}_{2}$-Besteuerung und hat mit einem Emissionshandel nichts zu tun, sondern ist politischer „Etikettenschwindel“ (Schnellenbach 2020, S. 38). Den Politikern scheint der Mut zu fehlen, die $\mathrm{CO}_{2}$ Bepreisung zum zentralen Leitinstrument für den Klimaschutz zu machen, die der Sachverständigenrat eindringlich gefordert hatte (SVR 2019; vgl. auch BMWi 2016). Im Gegenteil: Die kleinteiligen diskretionären Politikmaßnahmen werden die Lenkungswirkung des $\mathrm{CO}_{2}$-Preises beeinträchtigen und das Vertrauen der Bevölkerung in marktliche Prozesse vorhersehbar enttäuschen. Durch den jährlichen MonitoringProzess kommen planwirtschaftliche Sektorziele wieder ins Spiel, was einer politischen Bewertung der Marktergebnisse Tür und Tor öffnet. Damit ist zugleich ein hoher bürokratischer Aufwand für Beamte (in den Klima- und Energieabteilungen), Wissenschaftler und Berater verbunden, die sich in diese politische Bewertung möglicherweise auch mit eigenen Interessen - einbringen. Das sektorscharfe jährliche Zielmonitoring könnte eine gefährliche Eigendynamik in Gang setzen, die sich aus wachsenden Ansprüchen der Bevölkerung einerseits und Machbarkeitsillusionen der Politiker und der „Klimaschutzbürokratie“ andererseits speist und sich $\mathrm{zu}$ einer Interventionsspirale hochschaukelt. Es steht zu befürchten, dass dies zu einem weiteren Vertrauensverlust des marktwirtschaftlichen Gedankens führt. Die Diskreditierung des Europäischen Emissionshandelssystems hatte ja auch schon in der Vergangenheit der politischen Rhetorik der EEG-Lobbyisten und der Verfechter direkter staatlicher Eingriffe gedient - wenn man nicht in der Kritik mancher Politiker an den Schwankungen und an der Höhe des Zertifikatspreises ein Beleg dafür erblicken will, dass sie die Funktionsweise eines „Cap-and-trade“-Systems gar nicht verstanden haben (Weimann und Timme 2019). Schließlich impliziert ein niedriger Zertifikatspreis ja, dass es gelungen ist, $\mathrm{CO}_{2}$-Emissionen mit niedrigen Kosten zu vermeiden; dieser sollte deshalb eher ein Anlass zur Freude als ein Grund zur Kritik sein.

\subsection{Hindernisse auf dem Weg zu einer Reform}

Es gibt nicht nur die oben erwähnten politischen Gründe für eine diskretionäre Klimapolitik, sondern es existieren darüber hinaus auch Mechanismen, die die Abkehr von einer solchen Politik wesentlich erschweren.

So hat sich in den vergangenen Jahren ein „Wildwuchs“ unterschiedlicher Steuern, Abgaben und Umlagen auf verschiedene Formen des Energieverbrauchs her- 
ausgebildet, der einer effektiven und kosteneffizienten Klimapolitik im Weg steht (Wissenschaftlicher Beirat beim BMWI 2019, S. 4). Dieser Wildwuchs verzerrt die Investitionsentscheidungen von Haushalten und Unternehmen, führt zu hohen zusätzlichen Kosten und erbringt (fast) keine Fortschritte bei der Verringerung der THG- bzw. $\mathrm{CO}_{2}$-Emissionen (Bardt und Schaefer 2018; Frondel 2017). ${ }^{13}$ Dies hat die Politiker aber nicht dazu bewegt, die Klimapolitik im Allgemeinen und das EEG im Besonderen in Frage zu stellen bzw. grundlegend zu verändern. Auch die hohen Kosten des EEG haben zu keiner Reformbereitschaft der politisch Verantwortlichen und zu keinem grundlegenden Umsteuern in der Klimapolitik geführt.

Politiker versuchen, eine möglichst klare politische Haltung in bestimmten Politikbereichen einzunehmen, um sich so von Vertretern anderer politischer Parteien abzugrenzen. Diese politischen Positionierungen können dann nicht so leicht von Wissenschaftlern durch Aufklärungs- oder Informationsbemühungen beeinflusst werden, da im politischen Prozess meist „Haltung statt Wissen“ zählt. Änderungen in der einmal verkündeten politischen Positionierung von Abgeordneten sind daher im Regelfall nur sehr langsam und auf längere Sicht möglich. Politiker sind an die Wertund Richtungsentscheidungen ihrer Partei gebunden, die wiederum nur schrittweise (z.B. durch Parteitagsbeschlüsse) geändert werden können. Parteien sind dabei darauf bedacht, keine abrupten Kurswechsel zuzulassen, die möglicherweise ihre politische Geschlossenheit in Frage stellt oder gar Zweifel an der Zuverlässigkeit ihrer politischen Beschlüsse aufkommen lässt (Lindner 2005, S. 72-73). Dies schränkt zwangsläufig die Veränderungsbereitschaft und -fähigkeit der politischen Vertreter ein. Ein Politiker, der sich einmal öffentlich zugunsten des EEG positioniert hat, wird sich daher kaum von der nachgewiesenen Ineffizienz und Ineffektivität des EEG überzeugen lassen. So wird verständlich, warum das EEG auch nach 20 Jahren so ein enormes Beharrungsvermögen hat.

Eine grundlegende Reform der aktuellen Politik wird nicht nur durch diese Pfadabhängigkeiten erschwert, sondern auch durch deren scheinbare Erfolge. Zwischen dem politischen Versprechen eines wirksamen Klimaschutzes (und sogar der Klimaneutralität Deutschlands bis 2050) und den tatsächlichen Ergebnissen der Klimapolitik klafft eine enorme Anspruchslücke. Auf allen Ebenen formulieren Politiker immer ambitioniertere Ziele, die realistischer Weise kaum jemals erfüllt werden können. Um darüber hinwegzutäuschen, streben Politiker kleine, aber sichtbare Schritte an, damit sie darauf verweisen können, es gehe ja - zumindest ein wenig - in die richtige Richtung. In der aktuellen Klima- und Energiepolitik findet man zahlreiche Belege für diese politische Inszenierungsstrategie: Immer mehr Personal in den einschlägigen Behörden, mehr Gesetze und Verordnungen, zusätzliche Gremien und Abstimmungsrunden (das sogenannte „Klimakabinett“ der Bundesregierung) sowie ein umfassendes jährliches Monitoring-Wesen und mehr Öffentlichkeitsarbeit und Kampagnen (Kübler 2020, S. 41). Auch die starke Fokussierung von Politik und Öffentlichkeit auf den Stromsektor dient der politischen Selbstinszenierung und Selbstbestätigung. Denn hier erreicht der Anteil erneuerbarer Energien mittlerweile

${ }_{13}$ Die $\mathrm{CO}_{2}$-Emissionen in Deutschland sind in den vergangenen zehn Jahren fast unverändert geblieben (Dubbers et al. 2019). 
fast $50 \%$, was als vorzeigbarer „Erfolg“ ja offensichtlich die eigenen Bemühungen zu bestätigen scheint.

In diesem Sinne ist auch der sehr emotionale und moralisierende Grundton der gesellschaftlichen Klimadebatte in Deutschland zu sehen, mit dem das eigene klimapolitische Engagement gerechtfertigt und einer fachlich-objektiven Überprüfung entzogen werden soll. Die privaten und politischen Akteure profilieren sich in der Öffentlichkeit als „Klimaretter“ und kaschieren erfolgreich ihre privaten Interessen - ungeachtet hoher gesellschaftlichen Kosten und Ineffizienzen und regressiver Verteilungswirkungen. Kritische Stimmen, wie z.B. die des Sachverständigenrats (SVR 2019) oder des Wissenschaftlichen Beirats beim BMWi (2019), werden nicht gehört.

\section{7 Ökonomische Rationalität versus politische Opportunität}

Die Bilanz der bisherigen Klimapolitik in Deutschland ist sehr ernüchternd: Die klimapolitischen Ziele werden weitgehend verfehlt, die gesamtwirtschaftlichen Kosten erscheinen demgegenüber sehr hoch, und eine grundlegende Reform ist nicht in Sicht. Die EU hat mit dem vorgelegten Green Deal eine großangelegte Strategie bis 2050 vorgelegt, die klimapolitisch wenig zielführend ist und sich wirtschaftlich als sehr kostenintensiv erweisen wird. Das Klimaproblem ist aber weder aus klimapolitischer noch aus wirtschaftspolitischer Sicht ohne eine rationale Klimapolitik zu bewältigen. Zentrale Voraussetzungen dafür sind Effektivität der Maßnahmen und Effizienz in der Umsetzung. Weder die deutsche Klimapolitik noch die der EU erfüllen diese Vorbedingungen.

Erforderlich ist daher erstens ein grundlegender Strategiewechsel der deutschen und der europäischen Klimapolitik: Wir sollten endlich die Klimapolitik als internationales Koordinationsproblem erkennen und einen wichtigen Beitrag zu einer international koordinierten Klimapolitik leisten, in der eine freiwillige wechselseitige Kooperation der souveränen Staaten und die schrittweise Institutionalisierung eines einheitlichen $\mathrm{CO}_{2}$-Preises im Mittelpunkt stehen. Eine effiziente Klimapolitik erfordert außerdem die Einbeziehung auch der negativen Emissionstechnologien mittels einer einheitlichen Subvention für die Entfernung von $\mathrm{CO}_{2}$ aus der Atmosphäre. Denn nur mit einem einheitlichen $\mathrm{CO}_{2}$-Emissions-Preis bzw. einer einheitlichen $\mathrm{CO}_{2}$-Absorptions-Subvention über alle Sektoren können die Produktivkräfte der Marktwirtschaft effektiv und effizient für den Klimaschutz eingesetzt werden.

Dazu ist es nicht notwendig, dass sich alle Länder einig sind, aber eine gewisse „kritische Masse“ ist auf jeden Fall erforderlich, damit die Klimaentwicklung spürbar beeinflusst werden kann. Dies wäre dann gegeben, wenn sich die wichtigsten Industrieländer zu einer Art „Klimaclub“ zusammenschließen würden. Wenn sich diese Länder auf ein gemeinsames Reduktionsziel und eine einheitliche $\mathrm{CO}_{2}-\mathrm{Be}$ preisung einigen, dann könnte eine sowohl effektive als auch effiziente Klimapolitik betrieben werden. Die anderen Länder könnten auch zum Beitritt zu diesem Club bewegt werden - nämlich durch die Androhung handelspolitischer Sanktionen, also von „Klimazöllen“ auf deren Exporte. Neben der „Zoll-Peitsche“ wäre es im Fall von 
Entwicklungsländern auch gerechtfertigt, über das „Zuckerbrot“ finanzieller Hilfen als Gegenleistung für ihren Beitritt nachzudenken.

Zweitens müssen sich die EU und Deutschland auf den schon jetzt unvermeidbaren Klimawandel vorbereiten und die entsprechenden Anpassungsmaßnahmen vornehmen. Dazu gehören der Waldumbau, die Verstärkung der Deiche an der Nordsee, eine Verbesserung des Hochwasserschutzes im Inland und die Installation von Klimaanlagen in Krankenhäusern und anderen öffentlichen Gebäuden. Solche und ähnliche Maßnahmen haben aus ökonomischer Sicht den großen Vorteil, dass sie zu $100 \%$ der eigenen Bevölkerung im Inland Nutzen stiften, die entsprechenden Ausgaben also nicht verschwendet werden.

Drittens sollte die Erforschung und Entwicklung sowohl klimafreundlicher als auch verlässlicher und kostengünstiger Energiequellen und industrieller Produktionsverfahren vorangetrieben werden. Wenn wir durch stärkere technologische Forschung und Entwicklung attraktive Substitute für fossile Energiequellen gewinnen, werden die fossilen Rohstoffe ökonomisch an Wert verlieren und ungenutzt im Boden verbleiben. Das größte langfristige Potenzial hat hier sicher die Kernfusionsenergie.

Viertens ist die Klimapolitik der EU und Deutschlands unbedingt zu reformieren. Vollkommen unabhängig davon, welche Emissionsziele man sich setzt, lohnt es sich, die Kosteneffizienz des klimapolitischen Instrumentariums zu verbessern und auf symbolträchtige, aber ineffiziente, ja mitunter sogar ineffektive Maßnahmen zu verzichten. Dies lohnt sich natürlich umso mehr, je stärker die Treibhausgasemissionen reduziert werden sollen. Sollte doch noch eine international koordinierte Klimapolitik zustande kommen, müssen die EU und Deutschland das hierfür notwendige Instrumentarium zur Verfügung haben.

Es gibt also mehr als genug zu tun in der Klimapolitik - und zwar auch und gerade dann, wenn man die ökonomische Vernunft nicht über Bord wirft. Ob die notwendige Reform der Klimapolitik tatsächlich erfolgen und ob die ökonomische Vernunft mehr Beachtung finden wird, erscheint allerdings sehr fraglich. Denn die Handlungsanreize, denen die politischen Akteure gegenwärtig unterliegen, befördern gerade nicht eine ökonomisch rationale Politik, sondern eher das Gegenteil derselben. Politische Opportunität und ökonomische Rationalität stehen nicht im Einklang miteinander, sondern implizieren unterschiedliche, ja sogar gegensätzliche klimapolitische Strategien. Da aber nur eine Klimapolitik, die im Eigeninteresse der „politischen Unternehmer“ liegt, realisiert werden wird, muss davon ausgegangen werden, dass auch weiterhin die ökonomische Rationalität zugunsten der politischen Opportunität vernachlässigt werden wird. Dies wird sich nur bei einem grundlegenden Wandel der Anreizstruktur des politischen Systems ändern - oder aber, wenn die Sachzwänge so stark sind, dass man sich eine ökonomisch ineffiziente Klimapolitik nicht mehr leisten kann.

Möglicherweise wird es im Gefolge der aktuellen Corona-Krise dazu kommen. Denn die großen wirtschaftlichen Verluste, die diese Krise verursacht, die enorme Steigerung der Staatsausgaben und Staatsschulden infolge der Gegenmaßnahmen all das sollte Anlass genug sein, die bisherige Klimapolitik im Allgemeinen und den europäischen Green Deal im Besonderen auf den Prüfstand zu stellen. Zusätzliche Belastungen durch ineffektive und ineffiziente Maßnahmen zur Emissionsreduktion 
sind das letzte, was die europäische und die deutsche Wirtschaft jetzt brauchen können. Wenn die Corona-Krise dazu führt, dass in Zukunft eine ökonomisch und ökologisch rationale Klimapolitik betrieben und sich an der wirtschaftlichen und der naturwissenschaftlichen Realität - und nicht an Ideologie und Wunschdenken orientiert wird, so könnte man ihr doch noch etwas Gutes abgewinnen.

Im Moment sieht es allerdings (noch?) so aus, als ob die Corona-Krise im Gegenteil zur Fortsetzung der bisherigen Klimapolitik instrumentalisiert wird, da im Rahmen des Corona-Wiederaufbauprogramms „Next Generation EU“ der „Green Deal“" nicht etwa reformiert, sondern in seiner bisherigen Form gestärkt werden soll.

Funding Open Access funding enabled and organized by Projekt DEAL.

Open Access Dieser Artikel wird unter der Creative Commons Namensnennung 4.0 International Lizenz veröffentlicht, welche die Nutzung, Vervielfältigung, Bearbeitung, Verbreitung und Wiedergabe in jeglichem Medium und Format erlaubt, sofern Sie den/die ursprünglichen Autor(en) und die Quelle ordnungsgemäß nennen, einen Link zur Creative Commons Lizenz beifügen und angeben, ob Änderungen vorgenommen wurden.

Die in diesem Artikel enthaltenen Bilder und sonstiges Drittmaterial unterliegen ebenfalls der genannten Creative Commons Lizenz, sofern sich aus der Abbildungslegende nichts anderes ergibt. Sofern das betreffende Material nicht unter der genannten Creative Commons Lizenz steht und die betreffende Handlung nicht nach gesetzlichen Vorschriften erlaubt ist, ist für die oben aufgeführten Weiterverwendungen des Materials die Einwilligung des jeweiligen Rechteinhabers einzuholen.

Weitere Details zur Lizenz entnehmen Sie bitte der Lizenzinformation auf http://creativecommons.org/ licenses/by/4.0/deed.de.

\section{Literatur}

Bardt, H., \& Schaefer, T. (2018). Verteilungsprobleme und Ineffizienz in der Klimapolitik. IW-Kurzbericht Nr. 1. Köln: Institut der deutschen Wirtschaft.

Barrett, S. (1990). The problem of global environmental protection. Oxford Review of Economic Policy, 6(1), 68-79.

Barrett, S. (1994). Self-enforcing international environmental agreements. Oxford Economic Papers, 46(5), 878-894.

Bundesministerium für Umwelt, Naturschutz und nukleare Sicherheit (BMU) (2019). Klimaschutzprogramm 2030 der Bundesregierung zur Umsetzung des Klimaplans 2050. https://www.bundesregierung. de/resource/blob/975226/1679914/e01d6bd855f09bf05cf7498e06d0a3ff/2019-10-09-klima-massnahmendata.pdf. Zugegriffen: 28. April 2021.

Bundesministerium für Umwelt, Naturschutz und nukleare Sicherheit (BMU) (2020). Klimaschutz in Zahlen: Fakten, Trends und Impulse deutscher Klimapolitik. Berlin: BMU.

Bundesrechnungshof (2018). Bericht nach $\$ 99$ BHO über die Koordination und Steuerung zur Umsetzung der Energiewende durch das Bundesministerium für Wirtschaft und Energie. Bonn: Bundesrechnungshof.

Bundesregierung (2019). Klimaschutzprogramm 2030 beschlossen. https://www.bundesregierung.de/bregde/themen/klimaschutz/massnahmenprogramm-klima-1679498. Zugegriffen: 28. April 2021.

Bundesverband der Deutschen Industrie (BDI) (2018). Klimapfade für Deutschland. Berlin: BDI.

Bundesverband der Deutschen Industrie (BDI) (2020). Auswirkungen der Schließung von Kohlekraftwerken auf den deutschen Strommarkt. Berlin: BDI.

Cassel, S. (2017). Politische Ökonomie der Energiewende: Reformen des EEG im Interessengeflecht. In H. J. Thieme \& J. Haucap (Hrsg.), Ordnungsdefizite und Lösungsansätze (S. 37-58). Berlin: De Gruyter.

$\mathrm{CO}_{2}$-Abgabe (2019). Energiesteuern klima- \& sozialverträglich gestalten: Wirkungen und Verteilungseffekte des $\mathrm{CO}_{2}$-Abgabekonzepts auf Haushalte und Pendelnde. Freiburg: $\mathrm{CO}_{2}$-Abgabe eV. 
Crippa, M., et al. (2020). Fossil $\mathrm{CO}_{2}$ emissions of all world countries: JRC Report 2020. Luxemburg: Joint Research Centre.

Deutsche Energie-Agentur (2020). Klimaneutralität: Ein Konzept mit weitreichenden Implikationen. Berlin: Deutsche Energie-Agentur.

Dubbers, D., Stachel, J., Uwer, U. (2019). Energiewende: Warum sich Deutschland verrechnet. Münchner Merkur, 14. Okt., 3.

Edenhofer, O., \& Jakob, M. (2017). Klimapolitik. Ziele, Konflikte, Lösungen. München: Beck.

Edenhofer, O., Kalkuhl, M., \& Ockenfels, A. (2020). Das Klimaschutzprogramm der Bundesregierung: Eine Wende der deutschen Klimapolitik? Perspektiven der Wirtschaftspolitik, 21(1), 4-18.

Ehler, T. (2019). Deutsche Energiewende als „Rent-Seeking Paradise“? Energiewirtschaftliche Tagesfragen, 69(4), 43-45.

Europäische Kommission (2019). Der europäische Green Deal: Mitteilung der Kommission an das Europäische Parlament, den Europäischen Rat, den Rat, den Europäischen Wirtschafts- und Sozialausschuss und den Ausschuss der Regionen. https://ec.europa.eu/info/sites/info/files/european-greendeal-communication_de.pdf. Zugegriffen: 28. April 2021.

Farnung. R. (2021). Die großen Risiken der Energiewende. Münchner Merkur, 28. Jan., 6.

Frondel, M. (2017). Ineffektiv und ineffizient. Eine Bilanz der deutschen Klimapolitik. Potsdam-Babelsberg: Friedrich-Naumann-Stiftung für die Freiheit.

Intergovernmental Panel on Climate Change (2016). Klimaänderung 2014, Synthesebericht: Beitrag der Arbeitsgruppen I, II und III zum Fünften Sachstandsbericht des Zwischenstaatlichen Ausschusses für Klimaänderungen. Bonn: IPCC.

Kübler, K. (2020). Zu den Wirkungen des Klimaschutzprogramms 2030 - Eine picoökonomische Analyse. Energiewirtschaftliche Tagesfragen, 70(3), 36-41.

Lindner, C. (2005). Politik und Politikberatung - Versuch einer Verständigung. In U. Jens \& H. Romahn (Hrsg.), Glanz und Elend der Politikberatung (S. 67-83). Marburg: Metropolis.

Lomborg, B. (2020). False Alarm. New York: Basic Books.

Monopolkommission (2009). Sondergutachten 54: Strom und Gas: Energiemärkte im Spannungsfeld von Politik und Wettbewerb. Bonn: Monopolkommission.

Neubäumer, R. (2019). Der Klimawandel wird weitergehen - eine unbequeme Wahrheit. Wirtschaftsdienst, 99(11), 798-807.

Nordhaus, W. (2015). Climate clubs: overcoming free-riding in international climate policy. American Economic Review, 105(4), 1339-1370.

Nordhaus, W. (2019). Climate change: the ultimate challenge for economics. American Economic Review, 109(6), 1991-2014.

Pindyck, R. S. (2020). What we know and don't know about climate change, and implications for policy. NBER Working Paper 27304. Cambridge: National Bureau of Economic Research.

Pritzl, R. (2020). Realitätsillusion der deutschen Klimapolitik. Wirtschaftsdienst, 100(9), 701-706.

Quick, R. (2019). Eine $\mathrm{CO}_{2}$-Grenzsteuer oder ein Klimazoll? Wirtschaftliche Freiheit, 11. Oktober. http:// wirtschaftlichefreiheit.de/wordpress/?p=26067. Zugegriffen: 28. April 2021.

Rickels, W., et al. (2019). Welche Rolle spielen negative Emissionen für die zukünftige Klimapolitik? Perspektiven der Wirtschaftspolitik, 20(2), 145-158.

Sachverständigenrat zur Begutachtung der gesamtwirtschaftlichen Entwicklung (SVR) (2019). Aufbruch zu einer neuen Klimapolitik: Sondergutachten. Berlin: SVR.

Schnellenbach, J. (2020). Das Klimapaket der Bundesregierung: Dirigismus statt Preise. Wirtschaftswissenschaftliches Studium, 49(1), 37-39.

Sinn, H. W. (2008). Das grüne Paradoxon: Warum man das Angebot bei der Klimapolitik nicht vergessen darf. Perspektiven der Wirtschaftspolitik, 9(Sonderheft), 109-142.

Söllner, F. (1993). The greenhouse effect, property rights and developing countries. Zeitschrift für Wirtschafts- und Sozialwissenschaften, 113(2), 255-272.

Söllner, F. (2020). Der „Green Deal“ der EU: Ein umwelt- und wirtschaftspolitischer Irrweg. Wirtschaftliche Freiheit, 29. Juni. http://wirtschaftlichefreiheit.de/wordpress/?p=27578. Zugegriffen: 28. April 2021.

v. Storch, H., \& Krauß, W. (2013). Die Klimafalle. Die gefährliche Nähe von Politik und Klimaforschung. München: Hanser.

Techert, H., Niehues, J., \& Bardt, H. (2012). Ungleiche Belastung durch die Energiewende: Vor allem einkommensstarke Haushalte profitieren. Wirtschaftsdienst, 92(8), 507-512.

Von der Leyen, U. (2020). Lage der Union 2020. Rede gehalten am 16. September. https://ec.europa.eu/ info/strategy/strategic-planning/state-union-addresses/state-union-2020_de. Zugegriffen: 28. April 2021. 
Weimann, J. (2019). Der Ausstieg aus der Kohle: alternativlos oder verantwortungslos? Perspektiven der Wirtschaftspolitik, 20(1), 14-22.

Weimann, J. (2020). Elektroautos und das Klima: die große Verwirrung. Wirtschaftsdienst, 100(11), 890-895.

Weimann, J., \& Timme, F. (2019). Die Entfremdung des Emissionshandels. Energiewirtschaftliche Tagesfragen, 69(5), 29-32.

Wissenschaftlicher Beirat beim BMWA (2004). Zur Förderung erneuerbarer Energien. Berlin: Bundesministerium für Wirtschaft und Arbeit.

Wissenschaftlicher Beirat beim BMWi (2016). Die essentielle Rolle des $\mathrm{CO}_{2}$-Preises für eine effektive Klimapolitik. Berlin: Bundesministerium für Wirtschaft und Energie.

Wissenschaftlicher Beirat beim BMWi (2019). Energiepreise und effiziente Klimapolitik. Berlin: Bundesministerium für Wirtschaft und Energie. 